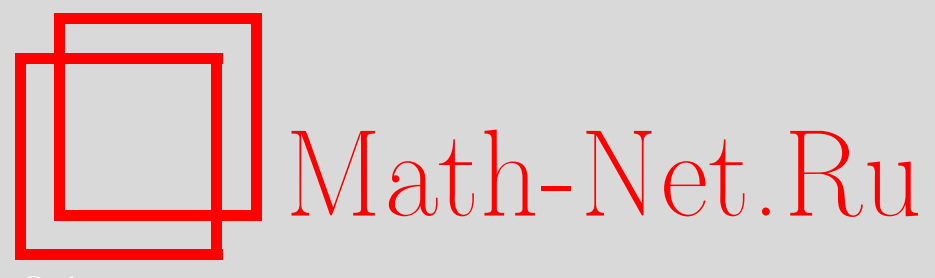

А. А. Нечаев, Д. А. Михайлов, Каноническая система образующих унитарного полиномиального идеала над коммутативным артиновым цепным кольцом, Дискрет. матем., 2001, том 13, выпуск 4, 3-42

DOI: https://doi.org/10.4213/dm307

Использование Общероссийского математического портала Math-Net.Ru подразумевает, что вы прочитали и согласны с пользовательским соглашением http: //www.mathnet.ru/rus/agreement

Параметры загрузки:

IP : 54.147 .182 .235

26 апреля 2023 г., 14:07:42 


\title{
Каноническая система образующих
} унитарного полиномиального идеала над коммутативным артиновым цепным кольцом

\author{
(C) 2001 г. А. А. Нечаев, Д. А. Михайлов
}

Пусть $R$ - коммутативное артиново цепное кольцо. Идеал $I$ кольца $\mathscr{R}_{k}=R\left[x_{1}, \ldots, x_{k}\right]$ называется унитарным, если факторкольцо $\mathscr{R}_{k} / I$ есть конечнопорожденный $R$-модуль. Для такого идеала построен стандартный базис, названный канонической системой образующих (KCO), сочетающий в себе хорошие свойства уже известной $\mathrm{KCO}$ идеала из $R[x]$ и базиса Гребнера полиномиального идеала над полем. В частности, используя $\mathrm{KCO}$, можно предложить алгоритм построения полной системы представителей $\mathscr{R}_{k}$ по модулю $I$, существенно более простой, чем алгоритм перебора, проверить, является ли факторкольцо $\mathscr{R}_{k} / I$ свободным $R$-модулем, и в случае, когда $R$ - конечное кольцо, указать явную формулу для числа $\left|\mathscr{R}_{k} / I\right|$, зависящую от числовых параметров КСО. С использованием КСО строится система образующих семейства $k$ линейных рекуррентных последовательностей с характеристическим идеалом $I$ и выводится критерий существования $k$-линейного регистра сдвига с этим характеристическим идеалом.

Работа выполнена при поддержке Российского фонда фундаментальных исследований, гранты 99-01-00941 и 99-01-00382.

\section{1. Введение}

Пусть $R$ - коммутативное артиново цепное кольцо, то есть коммутативное артиново локальное кольцо главных идеалов [1], $e-$ единица кольца $R$ и $\mathfrak{N}-$ его единственный максимальный идеал, индекс нильпотентности которого равен $n \in \mathbf{N}$. Тогда факторкольцо $\bar{R}=R / \mathfrak{N}$ есть поле, мультипликативная группа кольца $R$ имеет вид $R^{*}=R \backslash \mathfrak{N}$ и решетка его идеалов представляет собой строго убывающую цепочку

$$
R=\mathfrak{N}^{0} \triangleright \mathfrak{N} \triangleright \ldots \triangleright \mathfrak{N}^{n-1} \triangleright \mathfrak{N}^{n}=0 .
$$

Если $n=1$, то $R=\bar{R}-$ поле.

Идеал $I \triangleleft \mathscr{R}_{k}=R\left[x_{1}, \ldots, x_{k}\right]$ называется унитарным, если $R$-модуль $\mathscr{R}_{k} / I$ конечно порожден; эквивалентная формулировка: идеал $I \triangleleft \mathscr{R}_{k}=R\left[x_{1}, \ldots, x_{k}\right]$ называется унитарным, если $I$ содержит некоторую систему унитарных многочленов $F_{1}\left(x_{1}\right), \ldots, F_{k}\left(x_{k}\right)$ с коэффициентами из $R$ (систему элементарных унитарных многочленов). 
Известно (см. [5]), что любой идеал $I \subset \mathscr{R}_{k}$ имеет стандартный базис, то есть конечную систему образующих $\chi$ и некоторый алгоритм редуцирования (обобщенного деления с остатком) многочлена $H(\mathbf{x}) \in \mathscr{R}_{k}$ по модулю множества $\chi$, который позволяет за конечное число шагов проверять включение $H \in I$. В [21] приведен алгоритм построения такого базиса, учитывающий специфику кольца $R$. Получающийся при этом базис оказывается несократимым с точки зрения предложенного алгоритма проверки включения $H \in I$ и назван минимальным сильным базисом Гребнера. В общем случае для такого базиса получены лишь некоторые характеризации. Наиболее полно вид и свойства минимального сильного базиса Гребнера описаны только при $k=1$. В связи с этим необходимо отметить, что для $k=1$ данный базис был построен гораздо раньше в [20] (см. также $[3,16])$, где он был назван канонической системой образующих (КСО) полиномиального идеала. К сожалению, работа [21] не содержит ссылок на эти результаты. В [9] для случая, когда $R$ - кольцо Галуа [1], также построен стандартный базис (базис Гребнера) полиномиального идеала из $\mathscr{R}_{k}$. Базисы Гребнера из [9] и [21] не обладают рядом хороших свойств, которые имеет построенная нами КСО (подробнее см. раздел 5).

$\mathrm{B}[3,16,20]$ показано, что $\mathrm{KCO}$ идеала из $R[x]$ позволяет не только проверять включение $H \in I$ для многочлена $H(\mathbf{x}) \in \mathscr{R}_{k}$, но также вычислять мощность факторкольца $\mathscr{R}_{k} / I$, строить систему образующих семейства линейных рекуррент с характеристическим идеалом $I$, вычислять период идеала $I$ и так далее (см., в частности, теорему 2.1). Некоторыми такими хорошими свойствами обладает и базис Гребнера унитарного идеала над полем $[2,6,7,8,10,12]$. Отметим, что предложенный в [21] минимальный сильный базис Гребнера в случае $k>1$ описан недостаточно конструктивно для того, чтобы с его помощью можно было решать аналогичные задачи.

В данной работе на основе обобщения на случай $k>1$ известной теоремы Крулля [14] (см. ниже раздел 3) конструктивно строится каноническая система образующих (некоторый стандартный базис) унитарного идеала из $\mathscr{R}_{k}$, сочетающая в себе хорошие свойства КСО идеала из $R[x]$ и базиса Гребнера полиномиального идеала над полем.

\section{2. Основные предварительные результаты}

Здесь мы приводим результаты, ориентируясь на которые, будем строить названную КCO. Первый предварительный результат - описание и свойства КCO идеала в $R[x]$. Он представляет собой обобщение и развитие результатов работ $[13,15]$. При $n>1$ зафиксируем $\pi \in \mathfrak{N} \backslash \mathfrak{N}^{2}$. Тогда для любого $t \in\{0,1, \ldots, n\}$ верно равенство $\mathfrak{N}^{t}=\pi^{t} R$. Определим норму элемента $\alpha \in R$, многочлена $F(\mathbf{x}) \in \mathscr{R}_{k}$ и множества $S \subseteq \mathscr{R}_{k}$, соответственно, равенствами (см. [20])

$$
\begin{aligned}
\|\alpha\| & =\max \left\{t \in\{0,1, \ldots, n\}: \alpha \in \mathfrak{N}^{t}\right\}, \\
\|F\| & =\max \left\{t \in\{0,1, \ldots, n\}: F \in \pi^{t} \mathscr{R}_{k}\right\}, \\
\|S\| & =\max \left\{t \in\{0,1, \ldots, n\}: S \subseteq \pi^{t} \mathscr{R}_{k}\right\} .
\end{aligned}
$$

Теорема 2.1 ([20]). Пусть $I$ - унитарный идеал кольца $R[x]$. Тогда для некоторого $t \leqslant n-1$ существуют системы целочисленных параметров

$$
m_{0}>\ldots>m_{t} \geqslant 0, \quad 0=a_{0}<a_{1}<\ldots<a_{t}<a_{t+1}=n,
$$


удовлетворяющих условиям

(с1) для любих $F(x) \in R[x] u s=0,1, \ldots, t$

$$
\left(F(x) \in I, \operatorname{deg} F(x)<m_{s}\right) \Rightarrow\left(\|F(x)\| \geqslant a_{s+1}\right),
$$

(с2) существует система из $t+1 \leqslant n$ унитарного многочлена

$$
F_{0}(x), \ldots, F_{t}(x) \in R[x], \quad \operatorname{deg} F_{s}(x)=m_{s}, \quad s=0,1, \ldots, t,
$$

такая, что I содержит многочлень

$$
G_{0}(x)=F_{0}(x), G_{1}(x)=\pi^{a_{1}} F_{1}(x), \ldots, G_{t}(x)=\pi^{a_{t}} F_{t}(x) .
$$

Любая система многочленов (2.3), удовлетворяющих условиям (с1), (с2), обладает также следующими свойствами.

(с3) $E c л и F(x) \in I u\|F\| \geqslant a_{s}, 0 \leqslant s \leqslant t$, mo $F(x) \in\left(G_{s}(x), \ldots, G_{t}(x)\right)$, и в частности, верно равенство $I=\left(G_{0}(x), \ldots, G_{t}(x)\right)$.

(c4) Ecлu $s \in\{0,1, \ldots, t\} u a_{s} \leqslant a<a_{s+1}, m o$

$$
\begin{aligned}
I \cap \pi^{a} R[x] & =\left(\pi^{a-a_{s}} G_{s}(x), G_{s+1}(x), \ldots, G_{t}(x)\right), \\
\left(I: \pi^{a}\right) & =\left(F_{s}(x), \pi^{a_{s+1}-a} F_{s+1}(x), \ldots, \pi^{a_{t}-a} F_{t}(x), \pi^{n-a}\right) .
\end{aligned}
$$

(с5) Любой многочлен $H(x) \in R[x]$ однозначно представляется в виде

$$
H(x)=Q_{0}(x) F_{0}(x)+\ldots+Q_{t}(x) F_{t}(x)+Q_{t+1}(x),
$$

где $\operatorname{deg} Q_{s} F_{s}<m_{s-1} \partial л я s=1, \ldots, t, \operatorname{deg} Q_{t+1}<m_{t}$, и включение $H \in I$ эквивалентно системе неравенств $\left\|Q_{s}(x)\right\| \geqslant a_{s} \partial \Omega я s=1, \ldots, t+1$.

(с6) Если $R$ - конечное колъцо и $\bar{R}=G F(q)$, то $S=\mathscr{R} / I-$ также конечное кольцо и

$$
|S|=q^{\left(m_{0}-m_{1}\right) a_{1}+\ldots+\left(m_{t-1}-m_{t}\right) a_{t}+m_{t} n} .
$$

В условиях теоремы 2.1 система образующих (2.3) идеала $I$ называется канонической системой образующих $(\mathrm{KCO})$ идеала $I$.

Второй предварительный результат относится к свойствам базиса Гребнера полиномиального идеала над полем. В этой связи напомним некоторые понятия, придерживаясь терминологии монографии [10] с некоторыми изменениями в обозначениях. Пусть $\preceq-$ произвольное мономиальное (допустимое) упорядочение на множестве $\mathbf{N}_{0}^{k}$, то есть линейное упорядочение такое, что $\mathbf{0} \preceq \mathbf{i}$ для каждого $\mathbf{i} \in \mathbf{N}_{0}^{k}$ и для любых $\mathbf{u}, \mathbf{s}, \mathbf{t} \in \mathbf{N}_{\mathbf{0}}^{k}$ условие $\mathbf{s} \preceq \mathbf{t}$ влечет $\mathbf{s}+\mathbf{u} \preceq \mathbf{t}+\mathbf{u}$. Важными примерами мономиальных упорядочений на $\mathbf{N}_{0}^{k}$ являются лексикографическое упорядочение и градуированное лексикографическое упорядочение, которые задаются следующим образом: для произвольных

$$
\mathbf{i}=\left(i_{1}, \ldots, i_{k}\right), \mathbf{j}=\left(j_{1}, \ldots, j_{k}\right) \in \mathbf{N}_{0}^{k}
$$

соотношение $\mathbf{i} \preceq \mathbf{j}$ выполняется тогда и только тогда, когда для лексикографического упорядочения первая ненулевая из разностей $j_{k}-i_{k}, \ldots, j_{1}-i_{1}$ положительна, 
а для градуированного лексикографического упорядочения положительна первая ненулевая из разностей $\left(j_{1}+\ldots+j_{k}\right)-\left(i_{1}+\ldots+i_{k}\right), j_{k}-i_{k}, \ldots, j_{1}-i_{1}$.

Заметим, что для любого мономиального упорядочения верна импликация

$$
\forall \mathbf{i}, \mathbf{j} \in \mathbf{N}_{\mathbf{0}}^{k} \quad(\mathbf{i} \leqslant \mathbf{j}) \Rightarrow(\mathbf{i} \preceq \mathbf{j}),
$$

где $\mathbf{i} \leqslant \mathbf{j}$ означает покоординатное неравенство.

Для $A \in \mathscr{R}_{k}$ и $\mathbf{i}=\left(i_{1}, \ldots, i_{k}\right) \in \mathbf{N}_{0}^{k}$ через $\operatorname{Cf}\left(A, \mathbf{x}^{\mathbf{i}}\right)$ обозначим коэффициент многочлена $A(\mathbf{x})$ при произведении степеней независимых переменных (терме) $\mathbf{x}^{\mathrm{i}}=x_{1}^{i_{1}} \ldots x_{k}^{i_{k}}$. Конечное множество

$$
\Im(A)=\operatorname{supp}(A)=\left\{\mathbf{i} \in \mathbf{N}_{0}^{k}: \operatorname{Cf}\left(A, \mathbf{x}^{\mathbf{i}}\right) \neq 0\right\}
$$

назовем носителем многочлена $A$. Относительно выбранного упорядочения $\preceq$ множество $\subseteq(A)$ имеет максимальный элемент, который называется мультистепенью многочлена $A$ и обозначается $\operatorname{mgg} A$. Определим старший моном $\operatorname{Hm}(A)$, старший коэффициент $\mathrm{Hc}(A)$ и старший член (терм) $\mathrm{Ht}(A)$ многочлена $A$, соответственно, равенствами

$$
\begin{aligned}
\operatorname{Ht}(A) & =\mathbf{x}^{\operatorname{mdg} A}, \\
\operatorname{Hc}(A) & =\operatorname{Cf}(A, \operatorname{Ht}(A)), \\
\operatorname{Hm}(A) & =\operatorname{Hc}(A) \operatorname{Ht}(A) .
\end{aligned}
$$

Многочлен $A \in \mathscr{P}_{k}$, удовлетворяющий условию $\mathrm{Hc}(A)=e$ назовем унитарным.

Пусть теперь $R=P$, где $P$ - поле и $\mathscr{P}_{k}=P\left[x_{1}, \ldots, x_{k}\right]-$ кольцо многочленов над $P$. Напомним в этой ситуации основные определения, связанные с понятием полиномиальной редукции. Будем говорить, что многочлен $A \in \mathscr{P}_{k}$ редуцируется к многочлену $H \in \mathscr{P}_{k}$ с помощью многочлена $G \in \mathscr{P}_{k}$, элемента $b \in P$ и вектора $\mathbf{i} \in \mathbf{N}_{0}^{k}$ и писать

$$
A \rightarrow{ }_{(G, b, \mathbf{i})} H,
$$

если $\operatorname{Cf}\left(A, \mathbf{x}^{\mathbf{i}} \mathrm{Ht}(G)\right)=b \operatorname{Hc}(G) \neq 0$ и $H=A-b \mathbf{x}^{\mathbf{i}} G$. Пусть $\chi=\left\{G_{1}(\mathbf{x}), \ldots, G_{l}(\mathbf{x})\right\}-$ некоторое непустое подмножество $\mathscr{P}_{k}$. Скажем, что многочлен $A \in \mathscr{P}_{k}$ редуцируется к многочлену $H \in \mathscr{P}_{k}$ по модулю множества $\chi$ и будем писать $A \rightarrow_{\chi} H$, если для некоторого $d \in \mathbf{N}$ существуют многочлены $G_{j} \in \chi, H_{j} \in \mathscr{P}_{k}$, элементы $b_{j} \in P$ и векторы $\mathbf{i}_{j} \in \mathbf{N}_{0}^{k}, j=1, \ldots, d$, такие, что

$$
A \rightarrow{ }_{\left(G_{1}, b_{1}, \mathbf{i}_{1}\right)} H_{1} \rightarrow\left(G_{2}, b_{2}, \mathbf{i}_{2}\right) H_{2} \rightarrow\left(G_{3}, b_{3}, \mathbf{i}_{3}\right) \cdots \rightarrow_{\left(G_{d}, b_{d}, \mathbf{i}_{d}\right)} H_{d}=H .
$$

Назовем многочлен $A$ нормальным по модулю множества $\chi$, если не существует такого многочлена $H$, что $A \rightarrow_{\chi} H$. Через $N(\chi)$ обозначим множество всех нормальных многочленов по модулю $\chi$. Заметим, что $0 \in N(\chi)$ для любого множества $\chi$. Многочлен $H$ называется нормальной формой многочлена $A$ по модулю $\chi$, если $H \in N(\chi)$ и либо $A=H$, либо $A$ редуцируется к $H$ по модулю множества $\chi$. Пусть $N F(A, \chi)$ - множество всех нормальных форм многочлена $A$ по модулю $\chi$. Отметим, что для любого $A \in \chi$ множество $N F(A, \chi)$ содержит 0.

Для любого многочлена $A \in \mathscr{P}_{k}$ множество $N F(A, \chi)$ не пусто. Более того, при фиксированном упорядочении $\preceq$ и фиксированной нумерации элементов множества $\chi$ существует конечный алгоритм $\rightarrow \chi$ редуцирования (деления с остатком) любого многочлена $A \in\left(\mathscr{P}_{k} \backslash N(\chi)\right)$ по модулю множества $\chi$, при котором $A \rightarrow_{\chi} H$, где 
$H \in N(\chi)$. Таким образом, с помощью алгоритма редуцирования, любой многочлен $A \in \mathscr{P}_{k}$ может быть представлен в виде

$$
A(\mathbf{x})=Q_{1}(\mathbf{x}) G_{1}(\mathbf{x})+\ldots+Q_{l}(\mathbf{x}) G_{l}(\mathbf{x})+H(\mathbf{x}),
$$

где $H \in N F(A, \chi)$.

Конечное множество $\chi \subset \mathscr{P}_{k}$ унитарных многочленов назовем редуцированной системой Гребнера (относительно мономиального упорядочения $\preceq$ ), если каждый многочлен $G \in \chi$ является нормальным по модулю множества $\chi \backslash\{G\}$ и для любого многочлена $A$ из идеала $I=\mathscr{P}_{k} \chi$ множество $N F(A, \chi)$ равно $\{0\}$. Такая система многочленов называется также редуцированным базисом Гребнера идеала $I$. Отметим, что последнее условие в определении базиса Гребнера эквивалентно условию $|N F(A, \chi)|=1$ для любого $A \in \mathscr{P}_{k}$. Любой ненулевой идеал $I \triangleleft \mathscr{P}_{k}$ содержит единственный (при заданном упорядочении $\preceq$ ) редуцированный базис Гребнера $[7,10]$.

В дополнение к приведенному общему комбинаторно-алгебраическому описанию свойств базиса Гребнера нам понадобится также его линейно-алгебраическая характеризация в случае, когда $I$ - унитарный идеал. Эта характеризация связана с понятием диаграмма Ферре и основана на результатах работы [19].

Конечное множество $\mathscr{F} \subset \mathbf{N}_{0}^{k}$ называется диаграммой Ферре, если для любых

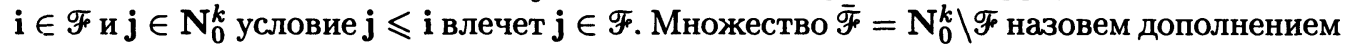
диаграммы Ферре $\mathscr{F}$. Обозначим $s$-ю строку единичной $k \times k$ матрицы над $\mathbf{Z}$ через $\mathbf{1}_{s}$. Назовем множество

$$
\mathscr{K} \mathscr{F}=\left\{\mathbf{i} \in \overline{\mathscr{F}}: \forall s=1, \ldots, k\left(i_{s}>0\right) \Rightarrow\left(\mathbf{i}-\mathbf{1}_{s} \in \mathscr{F}\right)\right\}
$$

множеством (внешних) углов диаграммы Ферре $\mathscr{F}$. Нетрудно увидеть, что КҒ можно охарактеризовать также как множество минимальных элементов частично упорядоченного множества $(\overline{\mathscr{F}}, \leqslant)$. Любая диаграмма Ферре $\mathscr{F}$ однозначно определяется своим множеством углов $\mathcal{H}:$

$$
\mathscr{F}=\mathbf{N}_{0}^{k} \backslash \bigcup_{\mathbf{i} \in \mathscr{K} \mathscr{F}}\left(\mathbf{i}+\mathbf{N}_{0}^{k}\right)
$$

Для любого $\mathbf{i} \in \mathscr{K}$ многочлен $G_{\mathbf{i}}(\mathbf{x})$ вида

$$
G_{\mathbf{i}}=\mathbf{x}^{\mathbf{i}}-\sum_{\mathbf{j} \in \mathscr{F}^{\prime}} g_{\mathbf{i}, \mathbf{j}} \mathbf{x}^{\mathbf{j}}
$$

называется $\mathscr{F}$-унитарным многочленом. Множество $\mathscr{F}$-унитарных многочленов $\chi=\left\{G_{\mathbf{i}}: \mathbf{i} \in \mathscr{K} F\right\}$ называется характеристическим множеством F्-унитарных многочленов. Важный крайний случай диаграммы Ферре: $\mathscr{F}=\varnothing-$ пустое множество, для него $\mathscr{F}=\{(0,0)\}$ и единственное характеристическое множество $\mathscr{F}$-унитарных многочленов есть $\chi=\{e\}$.

Заметим, что произвольному собственному идеалу $I \triangleleft \mathscr{P}_{k}$ соответствует факторкольцо $S=\mathscr{P}_{k} / I$ - кольцо операторов идеала $I$, которое можно представить в виде

$$
S=P\left[\vartheta_{1}, \ldots, \vartheta_{k}\right], \quad \vartheta_{s}=x_{s}+I, \quad s=1, \ldots, k .
$$

Кольцо $S$ есть $P$-алгебра, базис которой можно выбрать из элементов

$$
\boldsymbol{\vartheta}^{\mathbf{i}}=\vartheta_{1}^{i_{1}} \ldots \vartheta_{k}^{i_{k}}, \quad \mathbf{i} \in \mathbf{N}_{\mathbf{0}}^{k}
$$

причем, если $I-$ унитарный идеал, то этот базис конечен. 
Теорема 2.2. Пусть $I \triangleleft \mathscr{P}_{k}-$ унитарнъй идеал, $\preceq-$ мономиалъное отношение на $\mathbf{N}_{0}^{k}$. Тогда I имеет единственный редуцированный базис Гребнера $\chi$ относительно $\preceq$. Множество $\mathscr{K}=\{\operatorname{mdg} G: G \in \chi\}$ является множеством углов $\mathcal{K}=\mathscr{K} \mathscr{F}$ диаграммы Ферре

$$
\mathscr{F}=\mathscr{F}_{\chi}=\mathbf{N}_{0}^{k} \backslash \bigcup_{\mathbf{i} \in \mathscr{K}}\left(\mathbf{i}+\mathbf{N}_{0}^{k}\right)
$$

В случае, когда $I$ - собственный идеал, кольцо $S=\mathscr{P}_{k} / I$ операторов идеала I есть $P$-алгебра размерности $\left|\mathscr{F}_{\chi}\right|$ с базисом $\mathfrak{B}=\left\{\vartheta^{\mathbf{i}}: \mathbf{i} \in \mathscr{F}\right\}$.

Доказательство. Как уже отмечено, существование и единственность редуцированного базиса Гребнера доказаны, например, в [7, 10]. Мы приведем здесь лишь доказательство фактов, связанных с диаграммой Ферре, указав способ построения редуцированного базиса Гребнера, начинающийся с построения этой диаграммы и соответствующего базиса алгебры операторов.

В случае $I=\mathscr{P}_{k}$ утверждение теоремы очевидно. Пусть далее $I-$ собственный идеал кольца $\mathscr{P}_{k}$. Для каждого $\mathbf{j} \in \mathbf{N}_{0}^{k}$ положим

$$
\mathscr{F}(\mathbf{j})=\left\{\mathbf{i} \in \mathbf{N}_{0}^{k}: \mathbf{i} \prec \mathbf{j}\right\}, \quad \mathfrak{B}(\mathbf{j})=\left\{\boldsymbol{\vartheta}^{\mathbf{i}}: \mathbf{i} \in \mathscr{F}(\mathbf{j})\right\},
$$

где $\mathbf{i} \prec \mathbf{j}$ означает, что $\mathbf{i} \preceq \mathbf{j}$ и $\mathbf{i} \neq \mathbf{j}$. Назовем показатель $\mathbf{j}$ и элемент $\vartheta^{\mathbf{j}}$ критическими, если последний не является линейной комбинацией над $P$ элементов множества $\mathfrak{B}(\mathbf{j})$, и ординарными в противном случае. Пусть $\mathscr{F}=\mathscr{F}(I, \preceq)-$ множество всех критических показателей и $\mathfrak{B}=\left\{\boldsymbol{\vartheta}^{\mathbf{i}}: \mathbf{i} \in \mathscr{F}\right\}$. Нетрудно видеть, что $\mathfrak{B}-$ линейно независимая над $P$ система элементов. Покажем, что эта система - базис ${ }_{P} S$. Этот факт очевидно вытекает из следующего более сильного утверждения.

Лемма 2.1. Для любого ординарного показателя $\mathrm{s} \in \mathrm{N}_{0}^{k}$ элемент $\boldsymbol{\vartheta}^{\mathbf{s}}$ однозначно представляется в виде

$$
\boldsymbol{\vartheta}^{\mathbf{s}}=\sum_{\mathbf{j} \in \mathscr{F} \cap \mathscr{F}(\mathbf{s})} g_{\mathbf{s} \mathbf{j}} \boldsymbol{\vartheta}^{\mathbf{j}}, \quad g_{\mathbf{s} \mathbf{j}} \in P
$$

Доказательство. Используем индукцию по числу $о(\mathbf{s})$ ординарных показателей $\mathbf{i}$ таких, что $\mathbf{i} \prec \mathbf{s}$. Если $o(\mathbf{s})=0$, то все показатели из $\mathscr{F}(\mathbf{s})$ являются критическими и принадлежат $\mathscr{F}$, и так как по определению $\vartheta^{\mathbf{s}}$ линейно выражается через $\mathfrak{B}(\mathbf{s})$, верно (2.9). Пусть $l>0$ и утверждение верно для всех $\mathbf{s}$ таких, что $o(\mathbf{s})<l$. Рассмотрим случай, когда $o(\mathbf{s})=l$. По определению

$$
\boldsymbol{\vartheta}^{\mathbf{s}}=\sum_{\mathbf{i} \in \mathscr{F}(\mathbf{s})} c_{\mathbf{s i}} \vartheta^{\mathbf{i}}, \quad c_{\mathbf{s i}} \in P .
$$

Если в последней сумме $\mathbf{i}-$ критический показатель, то $\mathbf{i} \in \mathscr{F} \cap \mathscr{F}(\mathbf{s})$. Если $\mathbf{i}-$ ординарный показатель, то $o(\mathbf{i})<l$ и по предположению индукции $\vartheta^{\mathbf{i}}$ линейно выражается через систему элементов

$$
\left\{\boldsymbol{\vartheta}^{\mathbf{j}}: \mathbf{j} \in \mathscr{F} \cap \mathscr{F}(\mathbf{i})\right\} \subseteq\left\{\boldsymbol{\vartheta}^{\mathbf{j}}: \mathbf{j} \in \mathscr{F} \cap \mathscr{F}(\mathbf{s})\right\}
$$

Покажем, что $\mathscr{F}=\mathscr{F}(I, \preceq)-$ диаграмма Ферре. Конечность множества $\mathscr{F}$ следует из конечности размерности $p S$. Допустим, что $\mathbf{j} \in \mathscr{F}$ и $\mathbf{i} \leqslant \mathbf{j}$. Покажем, что $\mathbf{i} \in \mathscr{F}$. 
Если это не так, то $\vartheta^{\mathbf{i}}-$ ординарный элемент и для подходящих коэффициентов $c_{\mathbf{s}} \in P$ выполняется равенство

$$
\boldsymbol{\vartheta}^{\mathbf{i}}=\sum_{\mathbf{s} \prec \mathbf{i}} c_{\mathbf{s}} \vartheta^{\mathbf{s}}
$$

Пусть $\mathbf{u}=\mathbf{j}-\mathbf{i}$. Тогда $\mathbf{u} \in \mathbf{N}_{0}^{k}$, и ввиду мономиальности отношения $\preceq$ из условия $\mathbf{s} \prec \mathbf{i}$ следует $\mathbf{s}+\mathbf{u} \prec \mathbf{i}+\mathbf{u}=\mathbf{j}$. Отсюда, умножая обе части равенства (2.11) на $\boldsymbol{\vartheta}^{\mathbf{u}}$, получаем, что элемент $\boldsymbol{\vartheta}^{\mathbf{j}}$ является линейной комбинацией над $P$ элементов множества $\mathfrak{B}(\mathbf{j})$, что противоречит выбору показателя $\mathbf{j}$. Следовательно, $\mathbf{i} \in \mathscr{F}_{\text {и }} \mathscr{F}_{\mathscr{F}}$ - диаграмма Ферре.

Заметим теперь, что каждому представлению (2.9) соответствует унитарный (относительно $\preceq$ ) многочлен

$$
G_{\mathbf{s}}(\mathbf{x})=\mathbf{x}^{\mathbf{s}}-\sum_{\mathbf{j} \in \mathscr{F} \cap \mathscr{F}(\mathbf{s})} g_{\mathbf{s} \mathbf{j}} \mathbf{x}^{\mathbf{j}}=\mathbf{x}^{\mathbf{s}}-H_{\mathbf{s}}(\mathbf{x}),
$$

который, очевидно, принадлежит идеалу $I$. Рассмотрим множество $\mathscr{F}$ углов диаграммы $\mathscr{F}$ и покажем, что система многочленов

$$
\chi=\left\{G_{\mathbf{i}}(\mathbf{x}): \mathbf{i} \in \mathscr{K} \mathscr{F}\right\}
$$

есть редуцированный базис Гребнера идеала $I$. Пусть $I_{1}$ - идеал, порожденный всеми многочленами (2.12). Тогда $\mathscr{P}_{k} \chi \subseteq I_{1} \subseteq I$. Допустим, что существует многочлен

$$
A(\mathbf{x})=\sum_{\mathbf{s} \in \mathbf{N}_{0}^{k}} a_{\mathbf{s}} \mathbf{x}^{\mathbf{s}}
$$

принадлежащий $I \backslash I_{1}$. Так как $A$ сравним по модулю идеала $I_{1}$ с многочленом

$$
H(\mathbf{x})=\sum_{\mathbf{s} \in \mathbf{N}_{0}^{k}} a_{\mathbf{s}} H_{\mathbf{s}}(\mathbf{x})=\sum_{\mathbf{s} \in \mathscr{F}} h_{\mathbf{s}} \mathbf{x}^{\mathbf{s}},
$$

последний также принадлежит $I \backslash I_{1}$, что невозможно, поскольку $H(\vartheta)=0$ и ввиду линейной независимости системы $\mathfrak{B}$ также $H(\mathbf{x})=0$. Следовательно, $I=I_{1}$. Теперь для доказательства равенства

$$
I=\mathscr{P}_{k} \chi
$$

достаточно показать, что любой многочлен (2.12) принадлежит идеалу $\mathscr{P}_{k} \chi$. Для этого, очевидно, достаточно показать, что любой моном $\mathbf{x}^{\mathbf{s}}$ сравним по модулю этого идеала с многочленом, носитель которого принадлежит F्F. Назовем такой моном редуцируемым. Так как мономиальное отношение $\preceq$ является полным упорядочением, то есть любое непустое подмножество множества $\mathbf{N}_{0}^{k}$ содержит минимальный относительно $\preceq$ элемент (см., например, Следствие 6 Гл. 2 в [10]), последнее утверждение можно доказывать индукцией по $\preceq$. Если $\mathbf{s}=\mathbf{0}$, то утверждение очевидно. Пусть $\mathbf{0} \prec \mathbf{t}$ и для всех $\mathbf{s} \prec \mathbf{t}$ утверждение доказано, рассмотрим случай $\mathbf{s}=\mathbf{t}$. Если $\mathbf{s} \in \mathscr{F}$, то утверждение очевидно. Пусть $\mathbf{s} \in \overline{\mathscr{F}}$. Заметим, что $\mathscr{F}$ есть совокупность всех минимальных элементов решетки $(\overline{\mathscr{F}}, \leqslant)$. Следовательно, для подходящего $\mathbf{i} \in \mathscr{F}$ выполняются соотношения $\mathbf{i} \leqslant \mathbf{s}$ и $\mathrm{s}-\mathrm{i}=\mathbf{u} \in \mathrm{N}_{0}^{k}$. Из (2.12) (при $\mathrm{s}=\mathrm{i}$ ) следует, что

$$
\mathbf{x}^{\mathbf{i}} \equiv \sum_{\mathbf{j} \in \mathscr{F} \cap \mathscr{F}(\mathbf{i})} g_{\mathrm{ij}} \mathbf{x}^{\mathbf{j}} \quad\left(\bmod \mathscr{P}_{k} \chi\right)
$$


Умножая обе части этого сравнения на $\mathbf{x}^{\mathbf{u}}$, получаем, что

$$
\mathbf{x}^{\mathbf{s}} \equiv \sum_{\mathbf{j} \in \mathscr{F}_{\cap} \mathscr{F}_{(i)}} g_{\mathrm{ij}} \mathbf{x}^{\mathbf{j}+\mathbf{u}} \quad\left(\bmod \mathscr{P}_{k} \chi\right)
$$

Так как $\preceq$ - мономиальное отношение, в правой части последнего сравнения каждый показатель удовлетворяет условию $\mathbf{j}+\mathbf{u} \prec \mathbf{t}$, и по предположению индукции каждый моном редуцируем. Следовательно, редуцируем и моном $\mathbf{x}^{\mathbf{s}}$. Таким образом, равенство (2.14) доказано и $\chi-$ базис идеала $I$. Тот факт, что $\chi-$ редуцированный базис Гребнера и построенная диаграмма Ферре $\mathscr{F}_{(}(I, \preceq)$ есть диаграмма $\mathscr{F}_{\chi}$ из формулировки теоремы очевидно следует из описанных свойств входящих в $\chi$ многочленов.

Полезно отметить, что при введенных обозначениях многочлены $A(\mathbf{x}), H(\mathbf{x})$ связаны равенством

$$
A(\boldsymbol{\vartheta})=H(\boldsymbol{\vartheta})=\sum_{\mathbf{s} \in \mathcal{F}^{F}} h_{\mathbf{s}} \boldsymbol{\vartheta}^{\mathbf{s}}
$$

и $H$ есть нормальная форма многочлена $A$ по модулю базиса $\chi$. Таким образом, процесс редуцирования произвольного многочлена $A$ по модулю редуцированного базиса Гребнера $\chi$ эквивалентен процессу представления произвольного элемента $A(\vartheta) \in S$ в виде линейной комбинации элементов критического базиса $\mathfrak{B}$ алгебры ${ }_{P} S$.

В обозначениях теоремы 2.2 назовем диаграмму Ферре $\mathscr{F}_{\chi}=\mathscr{F}(I, \preceq)$ опорной диаграммой Ферре базиса $\chi$ и идеала $I$. Заметим, что $\chi$ есть характеристическое множество $\mathscr{F}_{\chi}$-унитарных многочленов и произвольный многочлен $H \in \mathscr{P}_{k}$ является нормальным по модулю множества $\chi$ в точности, если $\subseteq(H) \subseteq \mathscr{F}_{\chi}$.

Далее важно следующее утверждение.

Предложение 2.1. Если $I, J$ - унитарные идеаль колъца $\mathscr{P}_{k} u I \subseteq J$, то при биксированном мономиалъном упорядочении $\preceq$ справедливо включение

$$
\mathscr{F}(J, \preceq) \subseteq \mathscr{F}(I, \preceq)
$$

и равенство $I=J$ эквивалентно равенству $\mathscr{F}(J, \preceq)=\mathscr{F}(I, \preceq)$.

Доказательство. В случае $J=\mathscr{P}_{k}$ утверждение очевидно. Пусть далее $J \neq \mathscr{P}_{k}$. Рассмотрим

$$
T=\mathscr{P}_{k} / J=P\left[\gamma_{1}, \ldots, \gamma_{k}\right], \quad \gamma_{s}=x_{s}+J, \quad s=1, \ldots, k
$$

Из включения $I \subseteq J$ следует, что существует естественный эпиморфизм $P$-алгебр $\sigma: S \rightarrow T$ такой, что $\sigma\left(\vartheta_{s}\right)=\gamma_{s}, s=1, \ldots, k$. Отсюда следует, что любое соотношение $\sum c_{\mathbf{s}} \vartheta^{\mathbf{s}}=0$ индуцирует соотношение $\sum c_{\mathbf{s}} \gamma^{\mathbf{s}}=0$. Поэтому, если $\mathbf{j}-$ критический показатель в кольце $T$, то есть $\mathbf{j} \in \mathscr{F}(J, \preceq)$, то $\mathbf{j}-$ критический показатель и в кольце $S$, то есть $\mathbf{j} \in \mathscr{F}(I, \preceq)$. Равенство $\mathscr{F}(J, \preceq)=\mathscr{F}(I, \preceq)$ эквивалентно равенству $\operatorname{dim}_{P} T=\operatorname{dim}_{P} S$, которое эквивалентно равенству $\operatorname{Ker} \sigma=0$, то есть равенству $I=J$. 


\section{3. Обобщение теоремы Крулля}

Через $\bar{\alpha}, \bar{F}, \bar{S}$ обозначим образы элемента $\alpha \in R$, многочлена $F \in \mathscr{R}_{k}$ и подмножества $S \subseteq \mathscr{R}_{k}$ при соответствующих естественных эпиморфизмах

$$
R \rightarrow \bar{R}, \quad \mathscr{R}_{k} \rightarrow \bar{R}[\mathbf{x}] \cong \overline{\mathscr{R}}_{k}=\mathscr{R}_{k} / \pi \mathscr{R}_{k} .
$$

Результаты теоремы 2.1 основаны на хорошо известной теореме Крулля [14]: для любого многочлена $F(x) \in R[x]$ такого, что $\bar{F} \neq 0$, существуют унитарный многочлен $G(x) \in R[x]$ и обратимый многочлен $U(x) \in R[x]$ такие, что $G=U F$. Эту теорему можно сформулировать также и в следующем виде: для любого идеала $I \triangleleft R[x]$ такого, что $\bar{I} \neq \overline{0}$, существует унитарный многочлен $G(x) \in I$ такой, что $\bar{I}=\bar{R}[x] \bar{G}$.

Ниже приводится обобщение этого результата для кольца многочленов от $k>1$ переменных. Всюду далее предполагается, что на множестве $\mathbf{N}_{0}^{k}$ зафиксировано мономиальное упорядочение $\preceq$ и все редукции и базисы Гребнера строятся относительно $\preceq$.

Теорема 3.1. Пусть $I \triangleleft \mathscr{R}_{k}-$ унитарный идеал и $\Psi \subset I-$ система многочленов тахал, что $|\Psi|=|\bar{\Psi}| u \bar{\Psi}-$ редуцированнъй базис Гребнера идеала $\bar{I} \triangleleft \bar{\Re}_{k} c$

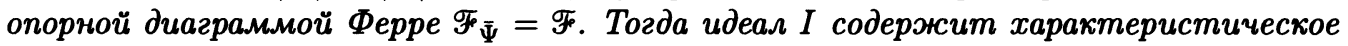
множество $\chi \subset I$ F-унитарных многочленов такое, что

$$
\bar{\chi}=\bar{\Psi} \text {. }
$$

Доказательство. Заметим, что существование исходной системы многочленов $\Psi \subset I$ и даже ее однозначная определенность по модулю идеала $\mathfrak{R} \mathfrak{R}_{k}$ (то есть однозначная определенность системы $\bar{\Psi}$ ) следуют из теоремы 2.2 .

Назовем многочлен $A \in \mathscr{R}_{k} \mathscr{F}$-унитарным по модулю $\pi^{s}$, если он представляется в виде $A=B+\pi^{s} C$, где $B, C \in \mathscr{R}_{k}$ и $B-\mathscr{F}$-унитарный многочлен.

Для доказательства существования системы $\mathscr{F}$-унитарных многочленов $\chi \subset I$ со свойством (3.1) достаточно указать алгоритм построения последовательности множеств

$$
\chi_{0}, \chi_{1}, \ldots, \chi_{t}, \ldots \subset I
$$

в которой $\chi_{t}-$ система $\mathscr{F}_{-}$-унитарных по модулю $\pi^{t+1}$ многочленов со свойством

$$
\bar{\chi}_{t}=\bar{\Psi} .
$$

Тогда $\chi=\chi_{n-1}-$ искомая система.

Пусть $\Psi=\left\{F_{1}(\mathbf{x}), \ldots, F_{l}(\mathbf{x})\right\}$. Тогда $\chi_{0}=\Psi$ - система F्ञ-унитарных по модулю $\pi$ многочленов, удовлетворяющая (3.3) при $t=0$. Допустим, что для некоторого $t<n-1$ уже построена система

$$
\chi_{t}=\left\{A_{1}(\mathbf{x}), \ldots, A_{l}(\mathbf{x})\right\} \subset I
$$

с нужными свойствами. Построим $\chi_{t+1}$. По предположению выполняются равенства

$$
\bar{A}_{s}(\mathbf{x})=\bar{F}_{s}, \quad A_{s}(\mathbf{x})=B_{s}(\mathbf{x})+\pi^{t+1} C_{s}(\mathbf{x}), \quad s=1, \ldots, l,
$$

где $B_{s}(\mathbf{x})-F_{F}$-унитарный многочлен. Так как (3.3) есть по условию редуцированный базис Гребнера над полем $\bar{R}$, любой многочлен $\bar{C}(\mathbf{x}) \in \overline{\mathscr{R}}_{k}$ имеет единственную 
нормальную форму по модулю $\bar{\chi}_{t}$, причем носитель многочлена $N F\left(\bar{C}(\mathbf{x}), \bar{\chi}_{t}\right)$ принадлежит $\mathscr{F}$. Это означает, что в кольце $\mathscr{R}_{k}$ существует представление

$$
C_{s}=\sum_{i=1}^{l} Q_{s, i}(\mathbf{x}) A_{i}+D_{s}(\mathbf{x}), \quad \Im\left(\bar{D}_{s}\right) \subseteq \mathscr{F}
$$

Положим $A_{s}^{\prime}(\mathbf{x})=B_{s}+\pi^{t+1} D_{s}, s=1, \ldots, l$. Последнее из условий (3.6) означает,

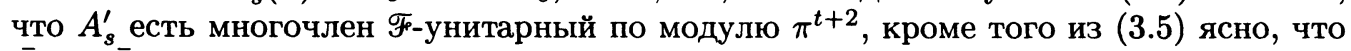
$\bar{A}_{s}^{\prime}=\bar{F}_{s}$. Наконец, из (3.5), (3.6) следует, что

$$
A_{s}^{\prime}=A_{s}-\pi^{t+1}\left(C_{s}-D_{s}\right)=A_{s}-\pi^{t+1} \sum_{i=1}^{l} Q_{s, i} A_{i} \in I
$$

Таким образом, $\chi_{t+1}=\left\{A_{1}^{\prime}, \ldots, A_{l}^{\prime}\right\}-$ искомая система.

Пусть $\mathscr{F} \subset \mathbf{N}_{0}^{k}-$ диаграмма Ферре. Будем говорить, что (при заданном мономи-

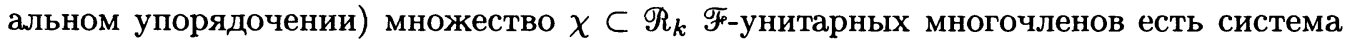
Крулля (круллева система) с опорной диаграммой Ферре $\mathscr{F}$, если множество $\bar{\chi}$ является редуцированной системой Гребнера с опорной диаграммой Ферре $\mathscr{F}_{\bar{\chi}}=\mathscr{F}_{\text {. }}$ Используя данное понятие, теорему 3.1 можно сформулировать следующим образом: при заданном мономиальном упорядочении для любого унитарного идеала $I \triangleleft \mathscr{R}_{k}$ существуют диаграмма Ферре $\mathscr{F} \subset \mathbf{N}_{0}^{k}$ и круллева система многочленов $\chi \subset I$ с опорной диаграммой $\mathscr{F}$ такие, что $\bar{I}=\overline{\mathscr{R}}_{k} \bar{\chi}$. Назовем эту систему главной частью канонической системы образующих (KCO) идеала $I$ (относительно упорядочения $\preceq$ ). Заметим, что такая система определяется идеалом $I$, вообще говоря, неоднозначно.

Например, если $k=1$, то любая круллева система состоит из одного унитарного многочлена, и в обозначениях теоремы 2.1 главная часть КСО унитарного идеала $I \in \mathscr{R}_{1}$ есть многочлен $F_{0}(x)$ или любой унитарный многочлен степени $m_{0}$ вида

$$
F_{0}(x)+\pi^{a_{1}} F_{1}(x) H_{1}(x)+\ldots+\pi^{a_{t}} F_{t}(x) H_{t}(x)
$$

Таким образом, в этом случае главная часть КCO унитарного идеала определена однозначно в точности, если это - главный идеал.

Отметим также, что если $R-$ не поле и $k>1$, то круллева система многочленов $\chi$, в отличие от редуцированной системы Гребнера $\bar{\chi}$, не обязательно состоит из унитарных многочленов.

Пример 3.1. Пусть $R=\mathrm{Z}_{4}$ и $\preceq-$ стандартное градуированное лексикографическое упорядочение на $\mathscr{R}_{2}$, то есть

$$
e \prec x_{2} \prec x_{1} \prec x_{2}^{2} \prec x_{1} x_{2} \prec x_{1}^{2} \prec \ldots
$$

Тогда система $\chi$, состоящая из многочленов

$$
G_{1}(\mathbf{x})=x_{1}^{3}, \quad G_{2}(\mathbf{x})=x_{1} x_{2}+2, \quad G_{3}(\mathbf{x})=x_{2}^{2}+2 x_{1}^{2}
$$

есть круллева система с опорной диаграммой $\mathscr{F}_{F}=\{(0,0),(0,1),(0,2),(1,0)\}$, соответствующая редуцированной системе Гребнера $\bar{\chi}=\left\{x_{1}^{3}, x_{1} x_{2}, x_{2}^{2}\right\} \subset \overline{\mathscr{R}}_{2}$, которая состоит из унитарных многочленов. Однако, $G_{3}(\mathbf{x})$ - не унитарный многочлен. 


\section{4. Каноническая система образуюццих унитарного идеала из $\mathscr{R}_{k}$}

Для построения указанной системы образующих мы определим редукцию (обобщенное полиномиальное деление) в $\mathscr{R}_{k}$ не стандартным способом. Для этого, наряду с понятиями старшей степени, старшего монома и старшего члена многочлена, мы введем ряд дополнительных понятий. Для произвольного многочлена $A \in \mathscr{R}_{k}$ назовем ведущей (мульти)степенью и обозначим через $\operatorname{ldg}(A)$ максимальный относительно $\preceq$ элемент $\mathbf{i} \in \subseteq(A)$, удовлетворяющий условию $\left\|\mathrm{Cf}\left(A, \mathbf{x}^{\mathbf{i}}\right)\right\|=\|A\|$. Если $R-$ поле, то параметры $\operatorname{ldg}(A)$ и $\operatorname{mdg} A$ совпадают. В общем же случае эти параметры связаны следующим образом. Ненулевой многочлен $A$ можно представить в виде $A=\pi^{\|A\|} A_{1}$, где $A_{1} \in \mathscr{R}_{k}, \bar{A}_{1} \neq \overline{0}$. Тогда $\operatorname{ldg}(A)=\operatorname{mdg} \bar{A}_{1} \preceq \operatorname{mdg} A$. Из описания круллевой системы многочленов вытекает следующее утверждение.

Предложение 4.1. Пусть $\mathscr{F} \subset \mathbf{N}_{0}^{k}-$ диаграмма Ферре, $\chi-$ круллева система F्-унитарных многочленов. Тогда справедливо равенство $\{\operatorname{ldg}(G): G \in \chi\}=\mathscr{K}$.

Определим ведущий терм, ведущий коэффициент и ведущий моном многочлена $A \in \mathscr{R}_{k}$, соответственно, равенствами

$$
\operatorname{Lt}(A)=\mathbf{x}^{\operatorname{ldg}(A)}, \quad \operatorname{Lc}(A)=\operatorname{Cf}\left(A, \mathbf{x}^{\operatorname{ldg}(A)}\right), \quad \operatorname{Lm}(A)=\operatorname{Lc}(A) \operatorname{Lt}(A) .
$$

По определению считаем, что $\operatorname{Lt}(\mathbf{0})=0, \operatorname{Lc}(\mathbf{0})=0$. Отметим, что многочлен $A$ является унитарным, если $\operatorname{ldg}(A)=\operatorname{mdg} A$ и $\operatorname{Lc}(A)=e$.

Заметим, что в случае, когда $R=P$ - поле, введенные здесь функции от $A$ совпадают с теми, которые были введены выше в параграфе 2 , то есть

$$
\operatorname{Lt}(A)=\operatorname{Ht}(A), \quad \operatorname{Lc}(A)=\mathrm{Hc}(A), \quad \operatorname{Lm}(A)=\operatorname{Hm}(A) .
$$

Определение 4.1 ( $L$-редукция на многочленах). Будем говорить, что многочлен $A$ редуцируется ( $L$-редуцируется) к многочлену $H \in \mathscr{R}_{k}$ с помощью многочлена $G \in \mathscr{R}_{k}$, элемента $b \in R$ и вектора $\mathbf{i} \in \mathbf{N}_{0}^{k}$ и писать $A \rightarrow(G, b, \mathbf{i}) H$, если

$$
\operatorname{Cf}\left(A, \mathbf{x}^{\mathrm{i}} \operatorname{Lt}(G)\right)=b \operatorname{Lc}(G) \neq 0, \quad H=A-b \mathbf{x}^{\mathrm{i}} G .
$$

Если при этом $\mathbf{x}^{\mathbf{i}} \operatorname{Lt}(G)=\mathbf{x}^{\mathbf{j}}$, то будем говорить также, что моном $a_{\mathbf{j}} \mathrm{x}^{\mathbf{j}}$ многочлена $A$ редуцируется ведущим мономом $\operatorname{Lm}(G)$ многочлена $G$.

Несложно доказать следующее утверждение.

Предложение 4.2. Если $A \rightarrow_{(G, b, \mathbf{i})} H, m o\|H\| \geqslant\|A\|$.

Определение 4.2. Пусть $\chi-$ некоторое непустое подмножество $\mathscr{R}_{k}$. Будем говорить, что многочлен $A \in \mathscr{R}_{k}$ редуцируется ( $L$-редуцируется) к многочлену $H \in \mathscr{R}_{k}$ по модулю множества $\chi$ и писать $A \rightarrow_{\chi} H$, если для некоторого $d \in \mathbf{N}$ существуют многочлены $G_{j} \in \chi, H_{j} \in \mathscr{R}_{k}$, элементы $b_{j} \in R$ и векторы $\mathbf{i}_{j} \in \mathbf{N}_{0}^{k}, j=1, \ldots, d$, такие, что

$$
A \rightarrow{ }_{\left(G_{1}, b_{1}, \mathbf{i}_{1}\right)} H_{1} \rightarrow{ }_{\left(G_{2}, b_{2}, \mathbf{i}_{2}\right)} H_{2} \rightarrow\left(G_{3}, b_{3}, \mathbf{i}_{3}\right) \cdots \rightarrow_{\left(G_{d}, b_{d}, \mathbf{i}_{d}\right)} H_{d}=H .
$$

Назовем многочлен $A$ нормальным по модулю множества $\chi$, если не существует такого многочлена $H$, что $A \rightarrow_{\chi} H$, то есть если многочлен $A$ не содержит такого ненулевого монома, который редуцируется ведущим мономом какого-либо многочлена из множества $\chi$. 
Через $N(\chi)$ обозначим множество всех нормальных многочленов по модулю $\chi$. Заметим, что $0 \in N(\chi)$ для любого множества $\chi$.

Предложение 4.3. Многочлен $A \in \mathscr{R}_{k}$ явллется нормалънъм по модулю непустого конечного множества $\chi \subset \mathscr{R}_{k}$ тогда и толъко тогда, когда для любых $\mathrm{i} \in \mathcal{S}(A)$ $u G \in \chi$ из неравенства $\operatorname{ldg}(G) \leqslant \mathbf{i}$ следует неравенство $\left\|\operatorname{Cf}\left(A, \mathbf{x}^{\mathbf{i}}\right)\right\|<\|\operatorname{Lc}(G)\|$.

Доказательство. Если моном $a_{\mathrm{i}} \mathrm{x}^{\mathrm{i}}$ многочлена $A$ редуцируется ведущим мономом многочлена $G \in \chi$, то $\operatorname{ldg} G \leqslant \mathbf{i}$ и $\left\|a_{\mathbf{i}}\right\|=\left\|\operatorname{Cf}\left(A, \mathbf{x}^{\mathbf{i}}\right)\right\| \geqslant\|\operatorname{Lc}(G)\|$. Поэтому, если $A$ удовлетворяет последнему набору условий предложения, то $A \in N(\chi)$.

Наоборот, допустим, что существуют $\mathbf{i} \in \Im(A), \mathbf{j} \in \mathbf{N}_{0}^{k}$ и $G \in \chi$ такие, что выполняются условия $\mathbf{i}=\operatorname{ldg}(G)+\mathbf{j}$ и $\left\|\operatorname{Cf}\left(A, \mathbf{x}^{\mathbf{i}}\right)\right\| \geqslant\|\operatorname{Lc}(G)\|$. Тогда

$$
\mathrm{Cf}\left(A, \mathbf{x}^{\mathbf{i}}\right)=\pi^{u} a, \quad \operatorname{Lc}(G)=\pi^{v} c
$$

где $a, c \in R^{*}, u \geqslant v$ и многочлен $A(\mathbf{x})$ редуцируется с помощью многочлена $G(\mathbf{x})$, вектора $\mathbf{j}$ и элемента $b=\pi^{u-v} a c^{-1}$, то есть $A(\mathbf{x}) \notin N(\chi)$.

Следствие 4.1. В условиях предложения (4.3) многочлен $A \in \mathscr{R}_{k}$ может бить редуцирован по модулю множества ұ тогда и только тогда, когда существуют $\mathbf{i} \in \mathbb{S}(A) u G \in \chi$ maxue, umo $\operatorname{ldg}(G) \leqslant \mathbf{i} u\|\operatorname{Lc}(G)\| \leqslant\left\|\operatorname{Cf}\left(A, \mathbf{x}^{\mathbf{i}}\right)\right\|$. Ecлu $\chi-$ круллева система многочленов с опорной диаграммой $\mathscr{F}_{\text {, }}$ то

$$
N(\chi)=\left\{A \in \mathscr{R}_{k}: \Im(A) \subseteq \mathscr{F}\right\}
$$

Многочлен $H$ назовем нормальной формой многочлена $A$ по модулю $\chi$, если $H \in N(\chi)$ и либо $A=H$, либо $A$ редуцируется к $H$ по модулю множества $\chi$. Пусть $N F(A, \chi)$ - множество всех нормальных форм многочлена $A$ по модулю $\chi$. Отметим, что для любого $A \in \chi$ множество $N F(A, \chi)$ содержит 0.

Теорема 4.1. Пусть $\chi=\left\{G_{1}, \ldots, G_{t}\right\} \subset \mathscr{R}_{k}-$ круллева система многочленов с опорной диаграммой Ферре $\mathscr{F} \subset \mathbf{N}_{0}^{k}$. Тогда существует алгоритм $\rightarrow \chi$ редуцирования многочленов $A \in \mathscr{R}_{k} \backslash N(\chi)$ по модулю множества $\chi$, при котором $A \rightarrow \chi H$, где $H \in N(\chi)$.

Доказательство. Произвольный многочлен $A \in \mathscr{R}_{k}$ однозначно представляется в виде

$$
A=A^{0}+A^{1}, \quad \Im\left(A^{0}\right) \subseteq \mathscr{F}, \quad \Im\left(A^{1}\right) \subset \bar{F}, \quad\left\|A^{1}\right\|=a \in\{0,1, \ldots, n\} .
$$

Основной стандартный шаг предлагаемого алгоритма состоит в следующем. Если $a=n$, то $A=A^{0} \in N(\chi)$. Если $a<n$, то многочлен $A^{1}$ представляется в виде

$$
A^{1}=\pi^{a} B(\mathbf{x}), \quad \subseteq(B) \subset \overline{\mathscr{F}}, \quad \bar{B}(\mathbf{x}) \neq \overline{0} .
$$

Далее нам потребуется следующая лемма.

Лемма 4.1. Пусть многочлены $B, G \in \mathscr{R}_{k} u h \in \overline{\mathscr{R}}_{k}$ maковъ, что $\bar{B} \rightarrow(\bar{G}, b, \mathbf{i})$ Тогда существует многочлен $H \in \mathscr{R}_{k}$ такой, что $\bar{H}=h u B \rightarrow\{G\} H$. 
Доказательство. По определению редукции существуют $b \in \bar{R}$ и $\mathbf{i} \in \mathbf{N}_{0}^{k}$ такие, что

$$
\operatorname{Cf}\left(\bar{B}, \mathbf{x}^{\operatorname{mdg} \bar{G}+\mathbf{i}}\right)=b \operatorname{Hc}(\bar{G}) \neq 0, \quad h=\bar{B}-b \mathbf{x}^{\mathbf{i}} \bar{G} .
$$

Так как $\|G\|=0$, то $\operatorname{Lc}(G) \in R^{*}, \operatorname{mdg} \bar{G}=\operatorname{ldg} G$ и $\operatorname{Hc}(\bar{G})=\overline{\operatorname{Lc}(G)}$. Положим

$$
c=\operatorname{Cf}\left(B, \mathbf{x}^{\operatorname{ldg} G+\mathrm{i}}\right) \operatorname{Lc}(G)^{-1}, \quad H=B-c \mathbf{x}^{\mathrm{i}} G .
$$

Тогда, по определению, $B \rightarrow{ }_{(G, c, \mathbf{i})} H$, причем $\bar{H}=h$, так как

$$
\bar{c}=\overline{\operatorname{Cf}\left(B, \mathbf{x}^{\operatorname{ldg} G+\mathbf{i}}\right) \operatorname{Lc}(G)^{-1}}=\operatorname{Cf}\left(\bar{B}, \mathbf{x}^{\operatorname{mdg} \bar{G}+\mathbf{i}}\right) \operatorname{Hc}(\bar{G})^{-1}=b .
$$

Вернемся к доказательству теоремы. Применяя к многочлену $\bar{B}$ над полем $\bar{R}$ алгоритм редуцирования по модулю системы Гребнера $\bar{\chi}$ (см., например, [2]), получаем, что

$$
\bar{B} \rightarrow_{\bar{\chi}} \bar{H}, \quad \Im(\bar{H}) \subseteq \mathscr{F} .
$$

Отсюда и из леммы 4.1 следует, что

$$
B \rightarrow_{\chi} H, \quad\left\|H^{1}\right\| \geqslant 1 .
$$

Тогда $A^{1}=\pi^{a} B \rightarrow_{\chi} \pi^{a} H$ и $A \rightarrow_{\chi} A^{0}+\pi^{a} H=A_{1}$, где $A_{1}^{1}=\pi^{a} H^{1}, \mid A_{1}^{1} \| \geqslant a+1$.

Таким образом, основной стандартный шаг алгоритма состоит в редуцировании многочлена $A$ по модулю множества $\chi$ к многочлену $A_{1}$, обладающему указанным выше свойством. Теперь доказательство существования нормальной формы многочлена $A$ и алгоритма ее построения легко завершается индукцией по параметру $n-a$.

Отметим, что в общем случае последнюю теорему нельзя дополнить утверждением о единственности нормальной формы. Соответственно неверно и утверждение о том, что идеалу $I=\mathscr{R}_{k} \chi$ принадлежат лишь многочлены с нулевой нормальной формой. Так, в примере $3.1 H(\mathbf{x})=2 x_{2}$ - ненулевой многочлен в нормальной форме, принадлежащий идеалу $I: H=2 G_{1}+x_{2} G_{2}-x_{1} G_{3}$.

Пример 4.1. Пусть $R=\mathbf{Z}_{4}$ и упорядочение $\preceq$ выбрано, как и в примере 3.1. Тогда система $\chi$, состоящая из многочленов

$$
G_{1}(\mathbf{x})=x_{1}^{2}-2, \quad G_{2}(\mathbf{x})=x_{1} x_{2}-2, \quad G_{3}(\mathbf{x})=x_{2}^{2}
$$

есть круллева система с опорной диаграммой

$$
\mathscr{F}_{F}=\{(0,0),(0,1),(1,0)\} .
$$

Многочлен $A=x_{1}^{2} x_{2}$ имеет две различные нормальные формы относительно $\chi$, именно, $2 x_{2}=A-x_{2} G_{1}$ и $2 x_{1}=A-x_{1} G_{2}$.

Условия, при которых обсуждаемая единственность нормальной формы имеет место, легко формулируются в терминах кольца операторов $T=\mathscr{R}_{k} / I$ идеала $I=R_{k} \chi$, которое мы можем рассматривать как $R$-модуль и представить как расширение факторкольца $\tilde{R}=R / I \cap R$ :

$$
T=\tilde{R}\left[\omega_{1}, \ldots, \omega_{k}\right], \quad \omega_{s}=x_{s}+I, \quad s=1, \ldots, k .
$$


Теорема 4.2. Пустъ $\chi \subset \mathscr{R}_{k}-$ круллева система с опорной диаграммой Ферре $\mathscr{F} \neq \varnothing$. Тогда $R$-модуль ${ }_{R} T$ имеет несократимую систему образующих

$$
\mathfrak{U}=\left\{\boldsymbol{\omega}^{\mathbf{i}}: \mathbf{i} \in \mathscr{F}\right\} .
$$

Для того, чтобы любой многочлен $A \in \mathscr{R}_{k}$ имел единственную нормальную форму по модулю $\chi$ достаточно, чтобы система $\mathfrak{A}$ была линейно независимой, то есть чтобъ модуль ${ }_{R} T$ был свободным.

Доказательство. Рассмотрим факторкольцо $S=\overline{\mathscr{R}}_{k} / \bar{I}$. Так как по условию $\bar{\chi}$ есть редуцированный базис Гребнера идеала $\bar{I}$, по теореме $2.2 S$ есть алгебра над полем $\bar{R}$ с базисом $\mathfrak{B}=\left\{\boldsymbol{\vartheta}^{\mathbf{i}}: \mathbf{i} \in \mathscr{F}\right\}$, где $\vartheta_{s}=x_{s}+\bar{I}, s=1, \ldots, k$.

Заметим, что отображение $\varphi: T \rightarrow S$ по правилу $\varphi(\tilde{A}(\boldsymbol{\omega}))=\bar{A}(\boldsymbol{\vartheta})$ есть корректно определенный эпиморфизм колец с ядром $\operatorname{Ker} \varphi=\pi T$. Действительно, $\varphi$ можно рассматривать как продолжение естественного эпиморфизма $\mu: \mathscr{R}_{k} \rightarrow T$ по правилу $\mu(A(\mathbf{x}))=\tilde{A}(\boldsymbol{\omega})$ с ядром $\operatorname{Ker} \mu=I$ до естественного эпиморфизма $\nu: \mathscr{R}_{k} \rightarrow S$ по правилу $\nu(A(\mathbf{x}))=\bar{A}(\boldsymbol{\vartheta})$ с ядром $\operatorname{Ker} \nu=I+\pi \Re_{k}$.

Так как Ker $\varphi$ - нильпотентный идеал, согласно лемме Накаямы (см., например, [1]) подмножество $M \subset T$ порождает модуль $R_{R} T$ тогда и только тогда, когда $\varphi(M)$ порождает модуль ${ }_{R} S$, то есть пространство ${ }_{R} S$. Так как $\varphi(\mathfrak{U})=\mathfrak{B}-$ базис ${ }_{\bar{R}} S$ и $\varphi$ инъективно на $\mathfrak{B}$, из сказанного следует первое утверждение теоремы.

Пусть теперь некоторый многочлен $A \in \mathscr{R}_{k}$ имеет две различные нормальные формы, скажем $H_{1}$ и $H_{2}$, по модулю системы $\chi$. Тогда $A(\boldsymbol{\omega})=H_{1}(\boldsymbol{\omega})=H_{2}(\boldsymbol{\omega})$ и $H=H_{1}-H_{2}-$ ненулевой многочлен с носителем $\subseteq(H) \subseteq \mathscr{F}$, удовлетворяющий равенству $H(\boldsymbol{\omega})=0$. Следовательно, система $\mathfrak{U}$ линейно зависима.

Отметим, что вопрос о необходимости последнего условия в теореме 4.2 остается открытым.

Наш основной результат составляет следующая теорема.

Теорема 4.3. Пустъ $I \triangleleft \mathscr{R}_{k}-$ унитарный идеал, $\preceq-$ мономиалъное упорядочение на $\mathbf{N}_{0}^{k}$. Тогда для некоторого $t \in\{0,1, \ldots, n-1\}$ существуют цепочка

$$
\mathscr{F}_{t} \subset \mathscr{F}_{t-1} \subset \ldots \subset \mathscr{F}_{0}
$$

строго упорядоченных по включению диаграмм Ферре и набор

$$
0=a_{0}<a_{1}<\ldots<a_{t}<a_{t+1}=n
$$

целых чисел, удовлетворяющие следующим условиям.

(C1) Для любах $F \in \mathscr{R}_{k} u s=0,1, \ldots, t$

$$
\left(F \in I, \subseteq(F) \subseteq \mathscr{F}_{s}\right) \Rightarrow\left(\|F\| \geqslant a_{s+1}\right) .
$$

(C2) Для каждого $s=0,1, \ldots, t$ существует круллева система $\chi_{s} \subset \mathscr{R}_{k}$ $\mathscr{F}_{s}$-унитарных многочленов такая, что I содержит множество $\pi^{a_{s}} \chi_{s}$.

Любая система многочленов

$$
\chi_{0}, \chi_{1}, \ldots, \chi_{t},
$$

удовлетворяющая условиям (C1), (C2), обладает такюе следующими свойствами. 
(С3) Если $F \in I u\|F\|=a$, где $a \geqslant a_{s}$, для некоторого $s \in\{0,1, \ldots, t\}$, то

$$
F \in \mathscr{R}_{k}\left(\pi^{a} \chi_{s} \cup \pi^{a_{s+1}} \chi_{s+1} \cup \ldots \cup \pi^{a_{t}} \chi_{t}\right),
$$

в частности, $I=\mathscr{R}_{k}\left(\chi_{0} \cup \pi^{a_{1}} \chi_{1} \cup \ldots \cup \pi^{a_{t}} \chi_{t}\right)$.

(C4) Ecлu $s \in\{0,1, \ldots, t\} u a_{s} \leqslant a<a_{s+1}$, mo

$$
\begin{aligned}
I \cap \pi^{a} \mathscr{R}_{k} & =\mathscr{R}_{k}\left(\pi^{a} \chi_{s} \cup \pi^{a_{s+1}} \chi_{s+1} \cup \ldots \cup \pi^{a_{t}} \chi_{t}\right), \\
\left(I: \pi^{a}\right) & =\mathscr{R}_{k}\left(\chi_{s} \cup \pi^{a_{s+1}-a} \chi_{s+1} \cup \ldots \cup \pi^{a_{t}-a} \chi_{t} \cup\left\{\pi^{n-a}\right\}\right) .
\end{aligned}
$$

(C5) Если $H(\mathbf{x}) \in \mathscr{R}_{k}$ и $H_{1}(\mathbf{x}), \ldots, H_{t+1}(\mathbf{x})$ - многочлены, построенные рекурсивно по правилу

$$
H_{1} \in N F\left(H, \chi_{0}\right), \quad H_{s+1} \in N F\left(H_{s}, \chi_{s}\right), \quad s=1, \ldots, t,
$$

то включение $H \in I$ эквивалентно системе условий

$$
\left\|H_{s}(x)\right\| \geqslant a_{s}, \quad s=1, \ldots, t+1 .
$$

(C6) $E c л и ~ \bar{R}=G F(q)$ u $\left|\mathscr{F}_{s}\right|=m_{s}$ для $s=0,1, \ldots, t$, то $S=\mathscr{R}_{k} / I-$ конечное колъцо и справедливо равенство (2.4).

В условиях теоремы 4.3 система образующих

$$
\chi=\chi_{0} \cup \pi^{a_{1}} \chi_{1} \cup \ldots \cup \pi^{a_{t}} \chi_{t}
$$

идеала $I$ называется канонической системой образующих (KCO) идеала $I$ (относительно допустимого упорядочения $\preceq$ ).

Доказателъство. Построим цепочки (4.5) и (4.6), удовлетворяющие условиям (C1), $(\mathrm{C} 2)$. Пусть $\chi_{0}=\left\{G_{1}^{(0)}, \ldots, G_{t_{0}}^{(0)}\right\}$ - главная часть КСО идеала $I-$ круллева система с опорной диаграммой $\mathscr{F}_{0}$ такая, что $\bar{\chi}-$ редуцированный базис Гребнера идеала $\bar{I}$. Обозначим через $\mathcal{N}_{1}$ множество всех нормальных форм по модулю $\chi_{0}$ всех многочленов из идеала $I$. Заметим, что $\left\|\mathcal{N}_{1}\right\|=a_{1}>0$, так как нормальная форма любого многочлена из $\bar{I}$ по модулю $\bar{\chi}$ равна 0 . Если $a_{1}=n$, то $I=\mathscr{R}_{k} \chi_{0}$ и условия (C1), (С2) выполняются при $t=0$.

Пусть $a_{1}<n$. Рассмотрим идеал $I_{1}$, порожденный в $\mathscr{R}_{k}$ множеством $\mathcal{N}_{1} \cup \pi^{a_{1}} \chi_{0}$. Очевидно, что $I_{1} \subseteq I \cap \pi^{a_{1} \mathscr{R}_{k}}$ и, по построению, идеал $I_{1}$ содержит любой многочлен $F \in I \cap \pi^{a_{1}} \mathscr{R}_{k}$, поскольку редуцирование многочлена $F$ по модулю системы $\chi_{0}$ дает соотношение

$$
F=Q_{1} G_{1}^{(0)}+Q_{t_{0}} G_{t_{0}}^{(0)}+H,
$$

где $Q_{1}, \ldots, Q_{t_{0}} \in \pi^{a_{1}} \mathscr{R}_{k}, H \in \mathcal{N}_{1}$. Следовательно,

$$
I_{1}=I \cap \pi^{a_{1}} \mathscr{R}_{k} .
$$

Таким образом, диаграмма $\mathscr{F}_{0}$ и система параметров $a_{0}<a_{1}$ удовлетворяют условиям (C1), (C2) при $s=0$. Допустим, что мы уже построили набор строго убывающих диаграмм Ферре

$$
\mathscr{F}_{0} \supset \mathscr{F}_{1} \supset \ldots \supset \mathscr{F}_{i}
$$


и набор строго возрастающих целых чисел

$$
0=a_{0}<a_{1}<\ldots<a_{i}<a_{i+1} \leqslant n,
$$

а также соответствующий набор круллевых систем

$$
\chi_{0}, \chi_{1}, \ldots, \chi_{i}
$$

такие, что условия (C1), (C2) выполняются для $s=0, \ldots, i$ и

$$
a_{i+1}=\left\|\left\{A \in I: \Im(A) \subseteq \mathscr{F}_{i}\right\}\right\| .
$$

Если в (4.16) $a_{i+1}=n$, то $t=i$, и нужный набор параметров и круллевых систем построен.

Пусть $a_{i+1}<n$. Очевидно, достаточно показать, что в этом случае можно добавить к каждой из цепочек (4.13)-(4.15) еще по одному звену с сохранением свойств (C1), (C2) и свойства, соответствующего (4.16). Для произвольного многочлена $A \in \mathscr{R}_{k}$ построим цепочку нормальных форм

$$
H_{0} \in N F\left(A, \chi_{0}\right), \quad H_{s} \in N F\left(H_{s-1}, \chi_{s}\right), \quad s=1, \ldots, i .
$$

Назовем $H_{s} s$-й нормальной формой многочлена $A$ относительно набора круллевых систем (4.15). Заметим, что так как для $s \in\{1, \ldots, i\}$ выполняются включения $\subseteq\left(H_{s}\right) \subseteq \mathscr{F}_{s}$ и справедливы свойства $(\mathrm{C} 1),(\mathrm{C} 2)$, для любого $A \in I$ выполняются соотношения $H_{s} \in I,\left\|H_{s}\right\| \geqslant a_{s+1}, s=0,1, \ldots, i$. Обозначим через $\mathcal{N}_{s}$ множество всех нормальных форм $H_{s}$ всех многочленов $A$ из идеала $I$. Очевидно, что $\left\|\mathcal{N}_{i}\right\|=$ $a_{i+1}$. Рассмотрим идеал $I_{i+1}$, порожденный в $\mathscr{R}_{k}$ множеством $\mathcal{N}_{i} \cup \pi^{a_{i+1}}\left(\chi_{0} \cup \ldots \cup \chi_{i}\right)$. Очевидно, что $I_{i+1} \subseteq I \cap \pi^{a_{i+1}} \mathscr{R}_{k}$ и, по построению, идеал $I_{i+1}$ содержит любой многочлен $F \in I \cap \pi^{a_{i+1}} \mathscr{R}_{k}$. Следовательно,

$$
I_{i+1}=I \cap \pi^{a_{i+1}} \mathscr{R}_{k} .
$$

Рассмотрим идеал $J_{i+1}=\left(I_{i+1}: \pi^{a_{i+1}}\right)$. Очевидно, это унитарный идеал, содержащий системы (4.15), поэтому ввиду предложения 2.1 главная часть $\chi_{i+1} \mathrm{KCO}$ идеала $J_{i+1}$ есть круллева система с опорной диаграммой $\mathscr{F}_{i+1}$, принадлежащей $\mathscr{F}_{i}$, удовлетворяющая условию $\pi^{a_{i+1}} \chi_{i+1} \subset I$. В случае $J_{i+1}=\mathscr{R}_{k}$ верно равенство $\mathscr{F}_{i+1}=\varnothing$ и $\mathscr{F}_{i+1} \subset \mathscr{F}_{i}-$ строгое включение. Пусть далее $J_{i+1} \neq \mathscr{R}_{k}$. Тогда, при сделанных предположениях можно утверждать, что множество $\mathcal{N}_{i+1}$ содержит многочлен $H=\pi^{a_{i+1}} B$ для некоторого многочлена $B \in \mathscr{R}_{k}$ такого, что $\bar{B} \neq \overline{0}$ и

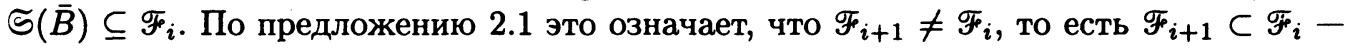
строгое включение. Таким образом, мы имеем строго убывающую цепочку диаграмм Фeppe

$$
\mathscr{F}_{0} \supset \mathscr{F}_{1} \supset \ldots \supset \mathscr{F}_{i+1}
$$

являющихся опорными, соответственно, для круллевых систем из набора

$$
\chi_{0}, \chi_{1}, \ldots, \chi_{i+1}
$$

удовлетворяющих условию (C2) для $s=0,1, \ldots, i+1$. Для каждого многочлена $A \in \mathscr{R}_{k}$ к цепочке нормальных форм (4.17) можно присоединить многочлен 
$H_{i+1} \in N F\left(H_{i}, \chi_{i+1}\right)$. При этом множество $\mathcal{N}_{i+1}$ всех $(i+1)$-х нормальных форм всех многочленов $A \in I$, имеет норму, большую, чем $a_{i+1}$, то есть параметр

$$
a_{i+2}=\left\|\left\{A \in I: \mathbb{S}(A) \subseteq \mathscr{F}_{i+1}\right\}\right\|
$$

удовлетворяет соотношениям $a_{i+1}<a_{i+2} \leqslant n$. Действительно, если $A \in I$ и справедливо включение $\subseteq(A) \subseteq \mathscr{F}_{i+1}$, то $\subseteq(A) \subseteq \mathscr{F}_{i}$ и потому $\|A\| \geqslant a_{i+1}$, то есть $A \in I_{i+1}$. Более того, $A=\pi^{a_{i+1}} B$, где $B \in J_{i+1}, \mathfrak{S}(B)=\subseteq(A) \subseteq \mathscr{F}_{i+1}$. Следовательно, $\|B\| \geqslant 1$ (ввиду выбора системы $\chi_{i+1}$ ) и $\|A\| \geqslant a_{i+1}+1$. Таким образом, мы доказали возможность удлинения цепочек (4.13)-(4.15) при условии $a_{i+1}<n$ и тем самым доказали существование набора параметров (4.5), (4.6) удовлетворяющих условиям (C1), (C2) теоремы.

Докажем теперь, что любая система многочленов (4.7), удовлетворяющая условиям (C1), (C2), обладает также свойствами (C3)-(C6).

Для проверки свойства (С3) используем индукцию по параметру $b=n-a \geqslant 0$. Если $b=0$, то $F=0$ и утверждение очевидно. Пусть $l>0$ и утверждение верно для всех $b<l$, докажем его для случая $b=l$. Многочлен $F$ можно представить в виде $F=\pi^{a} G, G \in \mathscr{R}_{k}$. Редуцируя многочлен $G$ по модулю системы $\chi_{s}=\left\{G_{1}^{(s)}, \ldots, G_{t_{s}}^{(s)}\right\}$, получаем равенство

$$
G=\sum_{1 \leqslant t \leqslant t_{s}} Q_{i} G_{i}^{(s)}+H, \quad \Im(H) \subseteq \mathscr{F}_{s} .
$$

Умножая обе части этого равенства на $\pi^{a}$ и пользуясь включением $\pi^{a} \chi_{s} \subset I$, получаем, что $\pi^{a} H=F_{1} \in I$, откуда, ввиду свойства (C1), следует неравенство $\left\|F_{1}\right\| \geqslant a_{s+1}$. Таким образом, $n-\left\|F_{1}\right\|<l$ и по предположению индукции справедливо включение

$$
\pi^{a} H=F_{1} \in \mathscr{R}_{k}\left(\pi^{a_{s+1}} \chi_{s+1} \cup \ldots \cup \pi^{a_{t}} \chi_{t}\right) .
$$

Отсюда и из (4.22) следует нужное включение для $F$.

Докажем справедливость свойства (C4). Равенство (4.8) есть переформулировка свойства (С3). Докажем (4.9). Если многочлен $G \in \mathscr{R}_{k}$ принадлежит идеалу из правой части (4.9), то $\pi^{a} G$ принадлежит, очевидно, идеалу из правой части (4.8). Следовательно, $\pi^{a} G \in I$ и $G \in\left(I: \pi^{a}\right)$. Наоборот, если $G \in\left(I: \pi^{a}\right)$, то $\pi^{a} G \in I \cap \pi^{a} \mathscr{R}_{k}$, то есть $\pi^{a} G$ принадлежит идеалу, стоящему в правой части равенства (4.8). Отсюда легко увидеть, что $G$ принадлежит правой части (4.9).

Докажем справедливость свойства (C5). При введенных обозначениях для подходящих многочленов $Q_{i}^{(s)} \in \mathscr{R}_{k}$ последовательные редукции из условия дают соотношения

$$
\begin{aligned}
H & =\sum_{i=1}^{t_{0}} Q_{i}^{(0)} G_{i}^{(0)}+H_{1}, \quad \Xi\left(H_{1}\right) \subseteq \mathscr{F}_{0}, \\
H_{s} & =\sum_{i=1}^{t_{s}} Q_{i}^{(s)} G_{i}^{(s)}+H_{s+1}, \quad \Im\left(H_{s+1}\right) \subseteq \mathscr{F}_{s} \quad s=1, \ldots, t .
\end{aligned}
$$

При этом, если выполняются условия (4.10), то $H_{t+1}=0$ и для подходящих многочленов $K_{i}^{(s)} \in \mathscr{R}_{k}$ выполняются равенства $Q_{i}^{(s)}=\pi^{a_{s}} K_{i}^{(s)}$. Поэтому, ввиду (C3),

$$
H=\sum_{s=0}^{t} \sum_{i=1}^{t_{s}} K_{i}^{(s)}\left(\pi^{a_{s}} G_{i}^{(s)}\right) \in I .
$$


Наоборот, если $H \in I$, то из (4.23) и (С3) следует, что $H_{1} \in I$, а из (4.23) и (C1) следует, что $\left\|H_{1}\right\| \geqslant a_{1}$. Аналогично рассуждая, далее из (4.24) и (C3), (C1) последовательно для $s=1, \ldots, t$ получаем, что в (4.24) выполняются соотношения

$$
\left\|Q_{i}^{(s)}\right\| \geqslant a_{s}, \quad Q_{i}^{(s)} G_{i}^{(s)} \in I, \quad H_{s+1} \in I, \quad\left\|H_{s+1}\right\| \geqslant a_{s+1} .
$$

Докажем теперь, что справедливо свойство (С7). Пусть $\bar{R}=G F(q)$ и $\left|\mathscr{F}_{i}\right|=m_{i}$ для $i=0,1, \ldots, t$. Для произвольного многочлена $A \in \mathscr{R}_{k}$ существует нормальная форма $H \in N F\left(A, \chi_{0}\right), \subseteq(H) \subseteq \mathscr{F}_{0}$, то есть многочлен $A$ сравним по модулю идеала $I$ с некоторым многочленом вида

$$
H=\sum_{\mathbf{i} \in \mathscr{F}_{0}} h_{\mathbf{i}} \mathbf{x}^{\mathbf{i}} .
$$

Заметим, что множество $\mathscr{H}$ всех многочленов (4.25) есть подгруппа мощности $|\mathscr{H}|=|R|^{|\mathscr{F}|}=q^{n m_{0}}$ аддитивной группы кольца $\mathscr{R}_{k}$, а множество $\mathscr{I}$ всех многочленов вида (4.25), принадлежащих идеалу $I$, есть подгруппа группы $\mathscr{H}$. Из сказанного ясно, что имеет место естественный изоморфизм аддитивных групп $S \cong \mathscr{H} / \mathscr{I}$. Следовательно,

$$
|S|=|\mathscr{H}| /|\mathscr{I}|=\frac{q^{n m_{0}}}{|\mathscr{I}|},
$$

и нам остается вычислить $|\varsubsetneqq|$. Для этого мы уточним критерий (С5) принадлежности многочлена $H$ вида (4.25) идеалу $I$ и получим метод перечисления таких многочленов.

Положим $H_{0}=H, H_{i} \in N F\left(H_{i-1}, \chi_{i}\right)$ для $i=1, \ldots, t$. Отметим, что $\Im\left(H_{i}\right) \subseteq \mathscr{F}_{i}$ и, согласно (C5), условие $H_{0} \in I$ равносильно системе неравенств (4.10). Заметим, что многочлен $H$ можно однозначно представить в виде

$$
\begin{aligned}
H & =H^{(0)}+H^{(1)}+\ldots+H^{(t)}, \\
\Im\left(H^{(j)}\right) & \subseteq \mathscr{F}_{j} \backslash \mathscr{F}_{j+1}, \quad j=0,1, \ldots, t, \quad \mathscr{F}_{t+1}=\varnothing .
\end{aligned}
$$

Соответственно, каждый из многочленов $H_{j}$ представляется в виде

$$
\begin{aligned}
H_{j} & =H_{j}^{(j)}+H_{j}^{(j+1)}+\ldots+H_{j}^{(t)}, \\
\Im\left(H_{j}^{(s)}\right) & \subseteq \mathscr{F}_{s} \backslash \mathscr{F}_{s+1}, \quad j=0,1, \ldots, t, \quad s=j, \ldots, t .
\end{aligned}
$$

Рассмотрим теперь систему многочленов

$$
N_{j} \in N F\left(H_{j-1}^{(j-1)}, \chi_{j}\right), j=1, \ldots, t .
$$

Каждый из этих многочленов также представляется в виде

$$
\begin{aligned}
N_{j} & =N_{j}^{(j)}+N_{j}^{(j+1)}+\ldots+N_{j}^{(t)}, \\
\Im\left(N_{j}^{(s)}\right) & \subseteq \mathscr{F}_{s} \backslash \mathscr{F}_{s+1}, \quad j=1, \ldots, t, \quad s=j, \ldots, t .
\end{aligned}
$$

При этом, как нетрудно увидеть, компоненты $H_{j}^{(s)}$ из (4.28) удовлетворяют равенствам

$$
\begin{array}{ll}
H_{1}^{(s)}=H_{0}^{(s)}+N_{1}^{(s)}, & s=1, \ldots, t, \\
H_{2}^{(s)}=H_{1}^{(s)}+N_{2}^{(s)}=H_{0}^{(s)}+N_{1}^{(s)}+N_{2}^{(s)}, & s=2, \ldots, t, \\
H_{j}^{(s)}=H_{j-1}^{(s)}+N_{j}^{(s)}=\ldots=H_{0}^{(s)}+N_{1}^{(s)}+\ldots+N_{j}^{(s)}, & s=j, \ldots, t, \quad j \geqslant 2 .
\end{array}
$$


Возможность перечисления и подсчета числа многочленов, принадлежащих группе $\mathscr{I}$, дает следующая лемма.

Лемма 4.2. Многочлен $H \in \mathscr{R}_{k}$ вида (4.25) принадлежит идеалу $I$, то есть подгруппе $\mathscr{I}$, тогда и толъко тогда, когда компоненты $H^{(s)}$ его разложения (4.27) $u$ последовательно получаемые из них многочлены (4.29) удовлетворяют следующей рекурсивной системе условий:

$$
\begin{aligned}
\left\|H^{(0)}\right\| & \geqslant a_{1}, \\
\left\|H^{(1)}+N_{1}^{(1)}\right\| & \geqslant a_{2}, \\
\left\|H^{(s-1)}+N_{1}^{(s-1)}+\ldots+N_{s-1}^{(s-1)}\right\| & \geqslant a_{s}, \quad s=2, \ldots, t, \\
H^{(t)}+N_{1}^{(t)}+\ldots+N_{t}^{(t)} & =0 .
\end{aligned}
$$

Доказательство. Пусть $H \in \mathscr{I}$. Тогда ввиду (C5) справедливы условия (4.10) и ввиду (4.28) выполняются неравенства $\left\|H_{j}^{(s)}\right\| \geqslant a_{j+1}$, из которых согласно (4.31)(4.33) следуют условия леммы.

Наоборот, пусть выполнены условия (4.34)-(4.37). Докажем (4.10). Из (4.34), используя (4.29) и предложение 4.2 , получаем, что $\left\|N_{1}\right\| \geqslant a_{1}$ (то есть $\left\|N_{1}^{(s)}\right\| \geqslant a_{1}$ для $s=1, \ldots, t)$. Отсюда, ввиду (4.35) и (4.31), получаем неравенства $\left\|H^{(1)}\right\| \geqslant a_{1}$ и $\left\|H_{1}^{(1)}\right\|=\left\|H^{(1)}+N_{1}^{(1)}\right\| \geqslant a_{2}$, последнее из которых, ввиду (4.29) и предложения 4.2, дает, в свою очередь, неравенство $\left\|N_{2}\right\| \geqslant a_{2}$. Таким образом, мы имеем системы неравенств

$$
\begin{aligned}
& \left\|H_{0}^{(0)}\right\| \geqslant a_{1}, \quad\left\|N_{1}\right\| \geqslant a_{1}, \\
& \left\|H_{0}^{(1)}\right\| \geqslant a_{1}, \quad\left\|H_{1}^{(1)}\right\| \geqslant a_{2}, \quad\left\|N_{2}\right\| \geqslant a_{2} .
\end{aligned}
$$

Теперь нетрудно увидеть, что из условий леммы и соотношений (4.28)-(4.33) индукцией по $s=0,1, \ldots, t$ доказываются неравенства

$$
\left\|H_{j}^{(s)}\right\| \geqslant a_{j+1}, \quad j=0,1, \ldots, s, \quad\left\|N_{s+1}\right\| \geqslant a_{s+1},
$$

из которых, согласно (4.28), следуют неравенства (4.10) и, ввиду (C5), включение $H \in \mathscr{I}$.

Теперь подсчитаем мощность группы $\mathscr{I}$, то есть количество многочленов $H \in \mathscr{R}_{k}$ с носителем, принадлежащим $\mathscr{F}_{0}$, удовлетворяющих условиям (4.34)-(4.37). Многочлен $H^{(0)}$, удовлетворяющий условию (4.34) с носителем, принадлежащим множеству $\mathscr{F}_{0} \backslash \mathscr{F}_{1}$ мощности $m_{0}-m_{1}$, можно выбрать $q^{\left(n-a_{1}\right)\left(m_{0}-m_{1}\right)}$ способами. Выбором $H^{(0)}$ из (4.29) однозначно определяется многочлен $N_{1}$. При фиксированном $N_{1}$ многочлен $H^{(1)}$, удовлетворяющий (4.35) с носителем, принадлежащим множеству $\mathscr{F}_{1} \backslash \mathscr{F}_{2}$ мощности $m_{1}-m_{2}$, можно выбрать $q^{\left(n-a_{2}\right)\left(m_{1}-m_{2}\right)}$ способами, и выбором $H^{(1)}$ из (4.29) однозначно определяется многочлен $N_{2}$. Продолжая аналогично далее, получаем, что для $s=1, \ldots, t$ при фиксированных $N_{1}, \ldots, N_{s-1}$ многочлен $H^{(s-1)}$, удовлетворяющий условию (4.36) с носителем, принадлежащим множеству $\mathscr{F}_{s-1} \backslash \mathscr{F}_{s}$ мощности $m_{s-1}-m_{s}$, можно выбрать $q^{\left(n-a_{s}\right)\left(m_{s-1}-m_{s}\right)}$ способами, и выбором $H^{(s-1)}$ из (4.29) однозначно определяется многочлен $N_{s}$. Наконец, при фиксированных $N_{1}, \ldots, N_{t}$, многочлен $H^{(t)}$, удовлетворяющий условию (4.37), определяется однозначно и его носитель, очевидно, принадлежит $\mathscr{F}_{t}$. Таким образом,

$$
|\mathscr{I}|=q^{\left(n-a_{1}\right)\left(m_{0}-m_{1}\right)+\left(n-a_{2}\right)\left(m_{1}-m_{2}\right)+\ldots+\left(n-a_{t}\right)\left(m_{t-1}-m_{t}\right)} .
$$


Теперь для доказательства равенства (2.4) достаточно воспользоваться равенством (4.26)

$$
|S|=q^{n m_{0}-\left(\left(n-a_{1}\right)\left(m_{0}-m_{1}\right)+\ldots+\left(n-a_{t}\right)\left(m_{t-1}-m_{t}\right)\right)}=q^{n m_{t}+a_{1}\left(m_{0}-m_{1}\right)+\ldots+a_{t}\left(m_{t-1}-m_{t}\right)}
$$

Теорема 4.3 доказана.

Следует заметить, что построенная нами $\mathrm{KCO}$, вообще говоря, не является редуцированным базисом Гребнера идеала $I$, поскольку она может содержать заведомо лишние для порождения идеала многочлены. Эта избыточность возникает в случае, если (в обозначениях теоремы 4.3) $t>0$ и для некоторого $s<t$ существует внешний угол $\mathbf{i} \in \mathscr{K}_{s}$, принадлежащий также $\mathscr{K}_{F_{s+1}}$. В таком случае существуют многочлены $G \in \chi_{s}, H \in \chi_{s+1}$ такие, что $\operatorname{ldg} G=\operatorname{ldg} H=\mathbf{i}$, и по построению $\bar{G}=\bar{H}$. Отсюда следует, что в системе образующих $\chi_{0} \cup \pi^{a_{1}} \chi_{1} \cup \ldots \cup \pi^{a_{t}} \chi_{t}$ идеала $I$ многочлен $\pi^{a_{s+1}} H$ является лишним.

Пример 4.2. Пусть $R=\mathbf{Z}_{4}$ и $I \triangleleft \mathscr{R}_{2}-$ идеал с системой образующих

$$
\psi=\left\{x_{1}^{2}, x_{2}^{2}, x_{1} x_{2}, 2 x_{2}\right\}
$$

и $\preceq-$ градуированное лексикографическое упорядочение, при котором $x_{2} \preceq x_{1}$.

Нетрудно увидеть, что главная часть КСО этого идеала есть круллева система $\chi_{0}=\left\{x_{1}^{2}, x_{2}^{2}, x_{1} x_{2}\right\}$ с опорной диаграммой

$$
\mathscr{F}_{0}=\{(0,0),(0,1),(1,0)\}
$$

и идеал $I_{1}=I \cap 2 \Re_{2}$ порождается многочленами $2 x_{1}^{2}, 2 x_{2}$. Следовательно, главная часть КСО идеала $J_{1}=\left(I_{1}: 2\right)$ есть круллева система $\chi_{1}=\left\{x_{1}^{2}, x_{2}\right\}$ с опорной диаграммой $\mathscr{F}_{2}=\{(0,0),(1,0)\}$. Нетрудно видеть, что получившиеся диаграммы Ферре имеют общий внешний угол $(2,0)$ и $2 x_{1}^{2}$ - лишний многочлен в $\mathrm{KCO} \chi_{0} \cup 2 \chi_{1}$ идеала $I$.

Отметим, однако, что именно эта допускаемая избыточность КСО позволяет сформулировать результаты пунктов (C3)-(С6) теоремы 4.3.

Результаты теорем 4.1 и 4.3 показывают, что представляет интерес изучение следующего понятия.

Определение 4.3. Круллеву систему многочленов $\chi \subset \mathscr{R}_{k}$ назовем системой Гребнера, если она есть КСО идеала $\mathscr{R}_{k} \chi$.

Предложение 4.4. Для собственного унитарного идеала $I \triangleleft \mathscr{R}_{k}$, имеющего $К С O$ $\chi$ вида (4.11), следующие условия эквивалентны:

(a) $t=0$, по есть $\chi_{0}$ есть система Гребнера, порождающая $I$;

(b) для каждого многочлена $A \in I$ выполняется равенство $N F\left(A, \chi_{0}\right)=0$;

(c) Факторхольцо $T=\mathscr{R}_{k} / I$ есть свободный $R$-модуль с базисом

$$
\mathfrak{U}_{0}=\left\{\omega^{\mathbf{i}}: \mathbf{i} \in \mathscr{F}_{0}\right\}
$$

определяемым из (4.3);

(d) факторкольцо $T=\mathscr{R}_{k} / I$ еств свободный $R$-модулъ. 
Дохазательство. Докажем, что (a) $\Rightarrow(\mathrm{b})$. Так как $\chi_{0}$ есть КСО идеала $I$, то в обозначениях теоремы $4.3 t=0$ и $a_{t+1}=a_{1}=n$. Поэтому, если $F \in N F\left(A, \chi_{0}\right)$, то $F \in I, S(F) \subseteq \mathscr{F}_{0}$ и по свойству (C1) $\|F\| \geqslant a_{1}=n$, то есть $F=0$.

Докажем, что (b) $\Rightarrow(\mathrm{c})$. Представим кольцо $T$ в виде (4.3). Если система образующих (4.38) - не базис ${ }_{R} T$, то существует многочлен $H \in \mathscr{R}_{k} \backslash 0$ такой, что $\widetilde{S}(H) \subseteq \mathscr{F}_{0}, H(\omega)=0$. В таком случае $H=N F(H, \chi), H \in I$ и $H \neq 0$, что противоречит условию (b).

Импликация (c) $\Rightarrow(d)$ очевидна.

Докажем, что $(\mathrm{d}) \Rightarrow(\mathrm{c})$. Пусть $\operatorname{dim}_{R} T=m$. Представим $T$ в виде (4.3) и рассмотрим факторкольцо $S=\overline{\mathscr{R}}_{k} / \bar{I}=\bar{R}[\vartheta], \vartheta_{s}=x_{s}+\bar{I}$. Как и при доказательстве теоремы 4.2 , можно утверждать, что отображение $\varphi: T \rightarrow S$ по правилу $\varphi(\tilde{A}(\boldsymbol{\omega}))=\bar{A}(\vartheta)$ есть корректно определенный эпиморфизм колец с нильпотентным ядром $\operatorname{Ker} \varphi=\pi T$. Отсюда по лемме Накаямы следует, что $\operatorname{dim}_{\bar{R}} S=m$.

Из определения $\mathrm{KCO}$ следует, что $\bar{\chi}_{0}-$ редуцированный базис Гребнера идеала $\bar{I}$. Отсюда по теореме 2.2 следует, что система $\mathfrak{B}_{0}=\left\{\vartheta^{\mathbf{i}}: \mathbf{i} \in \mathscr{F}_{0}\right\}$ есть базис пространства ${ }_{\bar{R}} S$. Следовательно, $\left|\mathscr{F}_{0}\right|=m$ и по лемме Накаямы система элементов (4.38) есть базис модуля ${ }_{R} T$ (так как $\varphi\left(\mathfrak{A}_{0}\right)=\mathfrak{B}_{0}$ и $\left.\left|\mathfrak{A}_{0}\right|=m\right)$.

Наконец, докажем, что (c) $\Rightarrow$ (a). При условии (c), если в системе (4.11) $t>0$, то $0<a_{1}<n, \chi_{1} \neq \varnothing$ и для любого $H \in \pi^{a_{1}} \chi_{1}$ выполняются условия

$$
H \neq 0, \quad \subseteq(H) \subseteq \mathscr{F}_{0}=\mathscr{F}_{F}, \quad H \in I .
$$

Отсюда следует, что $H(\omega)=0$ - нетривиальное линейное соотношение между элементами системы (4.38), что противоречит условию (c).

Представляется интересным вопрос: верно ли, что для любой системы Гребнера $\varphi \subset \overline{\mathscr{R}}_{k}$ существует система Гребнера $\chi \subset \mathscr{R}_{k}$ такая, что $\bar{\chi}=\varphi$, то есть можно ли поднять произвольную систему Гребнера $\varphi \subset \overline{\mathscr{R}}_{k}$ до системы Гребнера $\chi \subset \mathscr{R}_{k}$ ?

\section{5. КСО и известные базисы Гребнера}

Здесь мы сравним построенную КСО унитарного идеала с минимальным сильным базисом Гребнера из [21] и редуцированным базисом Гребнера из [9] и приведем пример, показывающий, что иногда КСО унитарного идеала существенно меньше указанных базисов.

\section{1. Минимальный сильный базис Гребнера}

В наших терминах сильный базис Гребнера из [21] определяется следующим образом.

Определение 5.1 (сильная $H$-редукция). Пусть $F \in \mathscr{R}_{k} \backslash\{0\}$ и $\psi \subset \mathscr{R}_{k} \backslash\{0\}-$ непустое подмножество.

Скажем, что $F$ сильно $H$-редуцируется за один шаг к многочлену $B \in \mathscr{R}_{k}$ по модулю множества $\psi$, если существует многочлен $G \in \psi$ такой, что $\operatorname{Hm}(G) \mid \operatorname{Hm}(F)$ и $B=F-m G$, где $m-$ моном, удовлетворяющий условию $\operatorname{Hm}(F)=m \operatorname{Hm}(G)$.

Скажем, что $F$ сильно $H$-редуцируется к $B$ по модулю множества $\psi$ и будем писать $F \mapsto \psi(B$, если $B$ получается из $F$ конечным числом сильных редукций за один шаг по модулю $\psi$. 
Теорема 5.1 ([21]). Пустъ $I$ - ненулевой идеал в $\mathscr{R}_{k} u \psi \subset I-$ конечное подмножество. Тогда следующие утверждения эквивалентны:

$$
\begin{aligned}
& \forall F \in \mathscr{R}_{k}(F \in I) \Rightarrow(F \mapsto \psi 0), \\
& \forall F \in \mathscr{R}_{k}(F \in I) \Longleftrightarrow(F \mapsto \psi 0) .
\end{aligned}
$$

Определение 5.2 (сильный базис Гребнера). Пусть $I \triangleleft \mathscr{R}_{k}-$ ненулевой идеал. Конечное подмножество $\psi \subset I \backslash\{0\}$ называется сильным базисом Гребнера идеала $I$, если оно удовлетворяет эквивалентным условиям теоремы 5.1. Сильный базис Гребнера $\psi$ называется минимальным, если никакое его собственное подмножество не является сильным базисом Гребнера идеала $\mathscr{R}_{k} \psi$.

Для многочлена $G \in \mathscr{R}_{k}$ его $A$-полиномом в [21] называется любой многочлен вида $b G$, где $b \in R$ - произвольный элемент такой, что $b R=\operatorname{Ann}(\operatorname{Hm}(G))$. Множество всех $A$-полиномов многочлена $G$ обозначается через $A P(G)$. Напомним, что $S$-полиномом многочленов $G_{1}, G_{2} \in \mathscr{R}_{k}$ со старшими мономами

$$
\operatorname{Hm}\left(G_{1}\right)=\pi^{t_{1}} a_{1} \mathbf{x}^{\mathbf{i}}, \quad \operatorname{Hm}\left(G_{2}\right)=\pi^{t_{2}} a_{2} \mathbf{x}^{\mathbf{j}},
$$

где $a_{1}, a_{2} \in R^{*}, \mathbf{i}=\left(i_{1}, \ldots, i_{k}\right), \mathbf{j}=\left(j_{1}, \ldots, j_{k}\right) \in \mathbf{N}_{0}^{k}$, называется многочлен

$$
S P\left(G_{1}, G_{2}\right)=\pi^{t_{1,2}-t_{1}} a_{1}^{-1} \mathbf{x}^{1-\mathbf{i}} G_{1}-\pi^{t_{1,2}-t_{2}} a_{2}^{-1} \mathbf{x}^{1-\mathbf{j}} G_{2},
$$

где $t_{1,2}=\max \left\{t_{1}, t_{2}\right\}, \mathbf{l}=\left(\max \left\{i_{1}, j_{1}\right\}, \max \left\{i_{2}, j_{2}\right\}, \ldots, \max \left\{i_{k}, j_{k}\right\}\right) \in \mathbf{N}_{0}^{k}$.

Следующая теорема дает характеризацию минимального сильного базиса Гребнера.

Теорема 5.2 ([21]). Конечное множество $\psi \subset \mathscr{R}_{k} \backslash\{0\}$ является минимальнъм силъным базисом Гребнера идеала $I=\mathscr{R}_{k} \psi$ в точности, если

(A) для каждого $G \in \psi$ существует $H \in A P(G)$ такой, что $H \mapsto{ }_{\psi} 0$

и для любых двух различных $G_{1}, G_{2} \in \psi$

(B) $S P\left(G_{1}, G_{1}\right) \mapsto{ }_{\psi} 0$,

(C) из условия $\mathrm{Ht}\left(G_{1}\right) \mid \operatorname{Ht}\left(G_{2}\right)$ следуют неравенства

$$
\operatorname{Ht}\left(G_{1}\right) \neq \operatorname{Ht}\left(G_{2}\right), \quad\left\|\mathrm{Hc}\left(G_{1}\right)\right\|>\left\|\mathrm{Hc}\left(G_{2}\right)\right\| \text {. }
$$

Приведем пример, показывающий, что в некоторых случаях КСО унитарного идеала существенно меньше минимального сильного базиса Гребнера.

Пример 5.1. Пусть $R=\mathbf{Z}_{4}, \preceq-$ чисто лексикографическое упорядочение множества $\mathbf{N}_{0}^{2}$, при котором $x_{1} \prec x_{2}$. Непосредственной проверкой легко показать, что множество

$$
\psi=\left\{G_{1}=x_{1}^{2}+3, G_{2}=x_{2}^{2}+3, G_{3}=2 x_{1}+2, G_{4}=2 x_{2}+x_{1}+1, G_{5}=x_{2} x_{1}+x_{2}+2\right\}
$$

удовлетворяет условиям (A)-(C) теоремы 5.2 и потому является минимальным сильным базисом Гребнера идеала $\mathscr{R}_{2} \psi$. Построим $\mathrm{KCO}$ этого идеала относительно отношения $\preceq$. 
Нетрудно видеть, что $\bar{I}=\overline{\mathscr{R}}_{2} \bar{\psi}=\overline{\mathscr{R}}_{2}\left(x_{2}^{2}+\overline{1}, x_{1}+\overline{1}\right)$, то есть множество $\bar{\chi}_{0}=\left\{\bar{G}_{2}, \bar{G}_{4}\right\}$ является редуцированным базисом Гребнера идеала $\bar{I}$ относительно отношения $\preceq$. Значит $\chi_{0}=\left\{G_{2}, G_{4}\right\}$ - главная часть КСО идеала $I$. Каждый из оставшихся многочленов первоначальной системы образующих $\psi$ идеала $I$ редуцируется к 0 по модулю системы $\chi_{0}$, так как

$$
G_{1}=\left(x_{1}+2 x_{2}+3\right) G_{4}, \quad G_{3}=2 G_{4}, \quad G_{5}=2 G_{2}+x_{2} G_{4} .
$$

Следовательно, $\chi_{0}$ есть КСО идеала $I$ и, очевидно, это - система образующих идеала $I$ наименьшей моџности. Опорная диаграмма Ферре найденной КСО есть, очевидно, $\mathscr{F}_{0}=\{(0,0),(0,1)\}$ и согласно теореме 4.3 (свойство (C6)) факторкольцо $S=\mathscr{R}_{k} / I$ имеет мощность $|S|=|R|^{2}=16$. Отметим также, что система $\chi_{0}$ вообще не является базисом Гребнера идеала $I$ в смысле [21], так как описанный в определении 5.1 алгоритм $H$-редуцирования не позволяет редуцировать к 0 по модулю $\chi_{0}$ многочлен $F=G_{1} \in I$. Действительно, при введенном упорядочении $\preceq$ справедливы равенства

$$
\operatorname{Hm}(F)=x_{1}^{2}, \quad \operatorname{Hm}\left(G_{2}\right)=x_{2}^{2}, \quad \operatorname{Hm}\left(G_{4}\right)=2 x_{2}
$$

и, очевидно, моном $\operatorname{Hm}(F)$ нельзя представить в виде линейной комбинации мономов $\operatorname{Hm}\left(G_{2}\right), \operatorname{Hm}\left(G_{4}\right)$.

\section{2. Редуцированный базис Гребнера}

Базис Гребнера из [9], построенный для случая, когда $R=G R\left(p^{m n}, p^{n}\right)-$ кольцо Галуа порядка $p^{m n}$ и характеристики $p^{n}$, можно определить в наших терминах следующим образом. Напомним, что каждый элемент $a \in R$ однозначно представляется в виде

$$
a=a_{0}+a_{1} p+\ldots+a_{n-1} p^{n-1}, \quad a_{0}, \ldots, a_{n-1} \in \Gamma=\left\{\alpha \in R: \alpha^{p^{m}}=\alpha\right\} .
$$

Такое представление $a$ называется его $p$-адическим разложением, а элемент $a_{i}$, $i=0,1, \ldots, n-1$, называется $i$-й координатой элемента $a$. Следуя [9], для $a \in R$ через $\eta_{p}(a)$ обозначим максимальный номер отличной от нуля координаты $p$-адического разложения элемента $a$.

Определение 5.3 ( $p$-редукция в $R$ ). Пусть $a, b, c \in R$ и $c \neq 0$. Будем говорить, что $a p$-редуцируется к $b$ по модулю $c$, если существует $d \in R$ такой, что $a=d c+b$ и либо $b=0$, либо $\eta_{p}(b)<\|c\|$. Элемент $a \in R$ назовем нормальным по модулю $c$, если $a$ не редуцируется по модулю $c$ ни к какому $b \in R \backslash\{a\}$.

Заметим, что элемент $b$ в этом определении однозначно определяется по $a$ и $c$ и имеет вид

$$
b=a_{0}+a_{1} p+\ldots+a_{\nu-1} p^{\nu-1}, \quad \nu=\|c\| .
$$

Нормальность $a$ по модулю $c$ означает, что $p$-адическое разложение $a$ имеет вид

$$
a=a_{0}+a_{1} p+\ldots+a_{\eta} p^{\eta}, \quad \eta<\|c\| .
$$

Определение 5.4 ( $Н p$-редукция на мономах). Пусть $\alpha, \beta, \gamma-$ мономы в $\mathscr{R}_{k}$ и $\gamma \neq 0$. Будем говорить, что $\alpha H p$-редуцируется к $\beta$ по модулю $\gamma$, если существуют $d \in R$ и терм $\mathbf{x}^{\mathbf{i}} \in \mathscr{R}_{k}$ такие, что

$$
\alpha=d \mathbf{x}^{\mathrm{i}} \gamma+\beta, \quad \operatorname{Ht}(\alpha)=\max \left\{\mathbf{x}^{\mathrm{i}} \operatorname{Ht}(\gamma), \operatorname{Ht}(\beta)\right\}
$$


и либо $\beta=0$, либо $\mathrm{Ht}(\beta)=\mathrm{Ht}(\alpha)$ и элемент $\mathrm{Hc}(\beta)$ является нормальным по модулю $\mathrm{Hc}(\gamma)$. Моном $\alpha \in \mathscr{R}_{k}$ назовем нормальным по модулю $\gamma$, если $\alpha$ не редуцируется по модулю $\gamma$ ни к какому моному $\beta \in \mathscr{R}_{k} \backslash\{\alpha\}$.

Определение 5.5 ( $Н p$-редукция на полиномах). Пусть $F, G, B \in \mathscr{R}_{k}$ и $G \neq 0$. Будем говорить, что $F H p$-редуцируется к $B$ по модулю $G$ за один шаг и писать $F \sim G B$, если в многочлене $F$ существует моном $\alpha \neq 0$, который редуцируется к некоторому моному $\beta$ по модулю монома $\gamma=\operatorname{Hm}(G)$ и для соответствующих $d \in R$, $\mathbf{x}^{\mathbf{i}} \in \mathscr{R}_{k}$, удовлетворяющих соотношениям из предыдущего определения, выполняется равенство $B=F-d \mathbf{x}^{\mathrm{i}} G$ (при этом обязательно $\left.\mathrm{Ht}(F)=\max \left\{\mathbf{x}^{\mathrm{i}} \mathrm{Ht}(G), \mathrm{Ht}(B)\right\}\right)$.

Будем говорить, что многочлен $F \in \mathscr{R}_{k} H p$-редуцируется к многочлену $B \in \mathscr{R}_{k}$ по модулю конечного множества $\psi \subset \mathscr{R}_{k}$ и писать $F \sim \psi B$, если $B$ может быть получен из $F$ путем конечной последовательности редукций в один шаг по модулю многочленов $G \in \psi$.

Многочлен $F \in \mathscr{R}_{k}$ называется нормальным по модулю $\psi$, если каждый его моном является нормальным по модулю $\mathrm{Hm}(G)$ для любого $G \in \psi$.

Определение 5.6. Пусть $I \triangleleft \mathscr{R}_{k}$. Конечное множество $\psi \subset I \backslash\{0\}$ называется базисом Гребнера идеала $I$, если для любого $F \in I$ существует $G \in \psi$ такой, что $\operatorname{Hm}(G) \mid \operatorname{Hm}(F)$. Базис Гребнера $\psi$ называется редуцированным, если никакое его собственное подмножество не является базисом Гребнера идеала $I=\mathscr{R}_{k} \psi$.

Теорема 5.3. Конечное множество многочленов $\psi=\left\{G_{1}, \ldots, G_{d}\right\} \subset \mathscr{R}_{k} \backslash\{0\} c$ множеством стариих коэфбициентов $\left\{\operatorname{Hc}\left(G_{j}\right)=p^{t_{j}} a_{j}: a_{j} \in R^{*}, j=1, \ldots, d\right\}$ является базисом Гребнера тогда и толъко тогда, когда выполняются следующие два условия:

(a) $S P\left(G_{i}, G_{j}\right) \sim \sim_{\psi} 0$ для всех различнъх $G_{i}, G_{j} \in \psi$,

(b) $p^{n-t_{j}} G_{j} \sim \psi 0$ для каљсдого $j=1, \ldots, d$.

Пусть $F, B, G \in \mathscr{R}_{k}$ и $G \neq 0$. Заметим, что из условия $F \mapsto_{G} B$ следует, что $F \sim G B$. Очевидно, что обратное утверждение для произвольных многочленов $F, B, G \in \mathscr{R}_{k}$ не верно. Отсюда следует, что если некоторое конечное множество многочленов $\psi \subset \mathscr{R}_{k} \backslash\{0\}$ удовлетворяет условиям (A) и (В) теоремы 5.2, то оно удовлетворяет также условиям (a) и (b) теоремы 5.3. Значит, над кольцом Галуа каждый минимальный сильный базис Гребнера является также редуцированным базисом Гребнера, и на систему многочленов $\psi$ из примера 5.1 можно смотреть также как на пример редуцированного базиса Гребнера в смысле работы [9].

Таким образом, конструктивно построенная в данной работе КСО унитарного идеала над локальным коммутативным артиновым цепным кольцом принципиально отличается от ранее известных минимального сильного базиса Гребнера ([21], раздел 5) и редуцированного базиса Гребнера ([9], раздел 5) и по сравнению с ними обладает дополнительными, привлекательными с прикладной точки зрения свойствами. Эти свойства КСО основаны, во-первых, на том, что каноническая система образующих строится не для произвольного, а только для унитарного полиномиального идеала и, во-вторых, на том, что для построения КСО вводится более эффективная, чем в [21], [9], процедура полиномиальной редукции: вместо $H$-редукции или $H p$-редукции (определения $5.1,5.5$ ) используется $L$-редукция (определение 4.1 ), основанная на более правильном, с нашей точки зрения, определении параметров $\mathrm{Lt}, \mathrm{Lc}, \mathrm{Lm}$ для многочленов над рассматриваемыми кольцами. 


\section{6. КСО и полилинейные регистры сдвига}

Пусть $R$ - коммутативное кольцо с единицей $e,{ }_{R} M-$ левый $R$-модуль. Понятие $k$-линейного (полилинейного) регистра сдвига над ${ }_{R} M$ определяется согласно [16] следующим образом.

Для $k \in \mathbf{N}$ произвольная функция $\mu: \mathbf{N}_{0}^{k} \rightarrow M$ называется $k$-последовательностью над $M$. Будем писать $\mu=\mu(\mathbf{z})$, где $\mathbf{z}=\left(z_{1} \ldots, z_{k}\right)$ - вектор независимых переменных на $\mathbf{N}_{0}^{k}$. Пусть $M^{\langle k\rangle}$ - множество всех $k$-последовательностей над $M$. Определим операцию умножения $k$-последовательности $\mu$ над $M$ слева на многочлен

$$
F(\mathbf{x})=\sum_{\mathbf{i} \in \mathbf{N}_{0}^{k}} f_{\mathrm{i}} \mathbf{x}^{\mathbf{i}} \in \mathscr{R}_{k}
$$

равенством

$$
F(\mathbf{x}) \mu=\nu, \quad \nu \in M^{\langle k\rangle}, \quad \nu(\mathbf{z})=\sum_{\mathbf{i} \in \mathbf{N}_{0}^{k}} f_{\mathbf{i}} \mu(\mathbf{z}+\mathbf{i}) .
$$

Таким образом, на $M^{\langle k\rangle}$ задается структура левого $\mathscr{R}_{k}$-модуля. Назовем $\mu \in M^{\langle k\rangle} k$ линейной рекуррентной последовательностью ( $k$-ЛРП), если существуют унитарные многочлены $F_{1}\left(x_{1}\right), \ldots, F_{k}\left(x_{k}\right) \in \mathscr{R}_{k}$ (от одной переменной), называемые элементарными, такие, что $F_{t}\left(x_{t}\right) \mu=0$ для любого $t=1, \ldots, k$.

Далее мы полагаем, что $R$ - артиново кольцо. В этом случае (и даже в случае, когда $R$ нетерово) последнее условие равносильно тому, что идеал

$$
\operatorname{An}(\mu)=\left\{F \in \mathscr{R}_{k}: F \mu=0\right\} \triangleleft \mathscr{R}_{k}
$$

является унитарным [16]. Для произвольного унитарного идеала $I \triangleleft \mathscr{R}_{k}$ левый $\mathscr{R}_{k}$-подмодуль $L_{M}(I)=\left\{\mu \in M^{\langle k\rangle}: I \mu=0\right\}$ модуля $M^{\langle k\rangle}$ называется $k$-ЛРП-семейством над $M$ с характеристическим идеалом $I$. Для произвольных $\mu \in M^{\langle k\rangle}$ и $\mathscr{F}=\left(\mathbf{i}_{1}, \ldots, \mathbf{i}_{d}\right) \subset \mathbf{N}_{0}^{k}$ положим $\mu[\mathscr{F}]=\left(\mu\left(\mathbf{i}_{1}\right), \ldots, \mu\left(\mathbf{i}_{d}\right)\right) \in M^{d}$. Скажем, что $\mu[\mathscr{F}]-$ диаграмма значений $k$-последовательности $\mu$ на полиэдре $\mathscr{F}$.

Пусть $I \triangleleft \mathscr{R}_{k}-$ унитарный идеал, $\mathscr{F} \subset \mathbf{N}_{0}^{k}-$ диаграмма Ферре. Пара $\langle I, \mathscr{F}\rangle$ называется $k$-линейным регистром сдвига ( $k$-ЛРС) или F्F-ЛРС над ${ }_{R} M$, если для любой функции с $\in M^{\mathscr{F}}$ существует единственная $k$-последовательность $\mu \in L_{M}(I)$ такая, что $\mu[\mathscr{F}]=\mathbf{c}[\mathscr{F}]$. Для данного ЛРС $\langle I, \mathscr{F}\rangle$ над ${ }_{R} M$ будем говорить, что диаграмма $\mathscr{F}$ - носитель, $I$ - характеристический идеал, а множество $L_{M}(I)$ - семейство выходных последовательностей ЛРС. Регистры сдвига называются эквивалентными, если множества их выходных последовательностей совпадают.

Следует отметить, что в [22], [23] и [24] для случаев $R=G F(q)$ и $R=Z_{m}$ изучалась конструкция $k$-LFSR, подобная рассматриваемой нами. Там же впервые была показана целесообразность использования стандартных базисов (базисов Гребнера) полиномиальных идеалов для построения $k$-LFSR.

Для рассматриваемого общего случая приведем доказательство анонсированного в [19] достаточного условия того, что пара из унитарного идеала и диаграммы Ферре образует $k$-линейный регистр сдвига. Напомним, что для произвольного идеала $I \triangleleft \mathscr{R}_{k}$ факторкольцо $T=\mathscr{R}_{k} / I$ представляется в виде (4.3)

$$
T=\tilde{R}\left[\omega_{1}, \ldots, \omega_{k}\right]
$$

где $\tilde{R}=R / I \cap R, \omega_{s}=x_{s}+I, s=1, \ldots, k$. 
Теорема 6.1 ([19]). Пустъ $I \triangleleft \mathscr{R}_{k}-$ унитарный идеал, $\mathscr{F} \subset \mathbf{N}_{0}^{k}-$ диаграмма Ферре и факторколъцо $T$ есть свободный $R$-модуль с базисом (4.4) $\mathfrak{A}=\left\{\boldsymbol{\omega}^{i}: \mathbf{i} \in \mathscr{F}\right\}$. Тогда пара $\langle I, \mathscr{F}\rangle$ образует $k$-линейный регистр сдвига над модулем ${ }_{R} M$.

Доказателъство. Покажем, что для произвольной функции с $\in M^{\mathscr{F}}$ существует $\mu \in L_{M}(I)$ такое, что $\mu[\mathscr{F}]=\mathbf{c}[\mathscr{F}]$. Так как $\mathfrak{A}-$ линейно независимая над $R$ система образующих модуля ${ }_{R} T$, для каждого $\mathbf{j} \in \mathbf{N}_{0}^{k}$ существует единственный набор коэффициентов $h_{\mathbf{s}}^{\mathbf{j}} \in R, \mathbf{s} \in \mathscr{F}$, такой, что

$$
\boldsymbol{\omega}^{\mathbf{j}}=\sum_{\mathbf{s} \in \mathscr{F}} h_{\mathbf{s}}^{\mathbf{j}} \boldsymbol{\omega}^{\mathbf{s}}
$$

Зададим $k$-последовательность $\mu \in M^{\langle k\rangle}$, полагая

$$
\mu(\mathbf{j})=\sum_{\mathbf{s} \in \mathscr{F}} h_{\mathbf{s}}^{\mathbf{j}} \mathbf{c}(\mathbf{s}), \quad \mathbf{j} \in \mathbf{N}_{0}^{k}
$$

Заметим, что $\mu[\mathscr{F}]=$ с. Покажем, что $\mu \in L_{M}(I)$. Рассмотрим произвольный многочлен

$$
F=\sum_{\mathbf{z} \in \mathbf{N}_{0}^{k}} f_{\mathbf{z}} \mathbf{x}^{\mathbf{z}} \in I
$$

Для произвольного $\mathrm{i} \in \mathbf{N}_{0}^{k}$ имеет место следующая цепочка равенств:

$$
(F \mu)(\mathbf{i})=\sum_{\mathbf{z} \in \mathbf{N}_{0}^{k}} f_{\mathbf{z}} \mu(\mathbf{i}+\mathbf{z})=\sum_{\mathbf{z} \in \mathbf{N}_{0}^{k}} f_{\mathbf{z}}\left(\sum_{\mathbf{s} \in \mathscr{F}} h_{\mathbf{s}}^{\mathbf{i}+\mathbf{z}} \mathbf{c}(\mathbf{s})\right)=\sum_{\mathbf{s} \in \mathscr{F}}\left(\sum_{\mathbf{z} \in \mathbf{N}_{0}^{k}} f_{\mathbf{z}} h_{\mathbf{s}}^{\mathbf{i}+\mathbf{z}}\right) \mathbf{c}(\mathbf{s})
$$

Несложно заметить, что

$$
\sum_{\mathbf{s} \in \mathscr{F}}\left(\sum_{\mathbf{z} \in \mathbf{N}_{0}^{k}} f_{\mathbf{z}} h_{\mathbf{s}}^{\mathbf{i}+\mathbf{z}}\right) \boldsymbol{\omega}^{\mathbf{s}}=\sum_{\mathbf{z} \in \mathbf{N}_{0}^{k}} f_{\mathbf{z}}\left(\sum_{\mathbf{s} \in \mathscr{F}^{F}} h_{\mathbf{s}}^{\mathbf{i}+\mathbf{z}} \boldsymbol{\omega}^{\mathbf{s}}\right)=\sum_{\mathbf{z} \in \mathbf{N}_{0}^{k}} f_{\mathbf{z}} \boldsymbol{\omega}^{\mathbf{i}+\mathbf{z}}=\boldsymbol{\omega}^{\mathbf{i}} F(\boldsymbol{\omega})=0 .
$$

Так как $\mathfrak{A}$ - линейно независимая система, из (6.4) следует, что

$$
\sum_{\mathbf{z} \in \mathbf{N}_{0}^{k}} f_{\mathbf{z}} h_{\mathbf{s}}^{\mathbf{i}+\mathbf{z}}=0
$$

для каждого $\mathrm{s} \in \mathscr{F}$. Отсюда и из (6.3) следует равенство $F \mu=0$.

Остается показать, что любая $k$-последовательность $\mu_{1} \in L_{M}(I)$, удовлетворяющая условию $\mu_{1}[\mathscr{F}]=\mu[\mathscr{F}]=\mathbf{c}[\mathscr{F}]$, совпадает с $\mu$. Выберем произвольно $\mathbf{j} \in \mathbf{N}_{0}^{k}$. Ввиду (6.1) многочлен

$$
F_{\mathbf{j}}=\mathbf{x}^{\mathbf{j}}-\sum_{\mathbf{s} \in \mathscr{F}} h_{\mathbf{s}}^{\mathbf{j}} \mathbf{x}^{\mathbf{s}}
$$

принадлежит идеалу $I$. Значит, $F_{\mathbf{j}} \mu_{1}=0$. В частности,

$$
\left(F_{\mathbf{j}} \mu_{1}\right)(\mathbf{0})=\mu_{1}(\mathbf{j})-\sum_{\mathbf{s} \in \mathscr{F}^{*}} h_{\mathbf{s}}^{\mathbf{j}} \mu_{1}(\mathbf{s})=0 .
$$

Отсюда и из условия $\mu_{1}[\mathscr{F}]=\mathbf{c}[\mathscr{F}]$ следует, что

$$
\mu_{1}(\mathbf{j})=\sum_{\mathbf{s} \in \mathscr{F}} h_{\mathbf{s}}^{\mathbf{j}} \mathbf{c}(\mathbf{s})=\mu(\mathbf{j})
$$


Напомним, что модуль ${ }_{R} M$ называется квазифробениусовым, или $Q F$-модулем, если для любых идеалов $I \triangleleft R$ и подмодулей $N \leqslant{ }_{R} M$, их аннуляторы

$$
A n_{M}(I)=\{\alpha \in M: I \alpha=0\}, \quad A n_{R}(N)=\{a \in R: a N=0\}
$$

удовлетворяют соотношениям

$$
A n_{R}\left(A n_{M}(I)\right)=I, \quad A n_{M}\left(A n_{R}(N)\right)=N .
$$

В случае, когда ${ }_{R} M-Q F$-модуль, верно обращение теоремы 6.1 для формулировки и доказательства которого нам потребуются следующие определения и результаты из [17].

Множество

$$
\Delta \mathscr{F}=\left\{\mathbf{i} \in \overline{\mathscr{F}} \mid \exists j \in\{1, \ldots, k\}: \mathbf{i}-\mathbf{1}_{j} \in \mathscr{F}\right\}
$$

называется границей диаграммы Ферре $\mathscr{F}$. Система многочленов вида

$$
\Phi=\left\{H_{\mathbf{r}}=\mathbf{x}^{\mathbf{r}}-\sum_{\mathbf{j} \in \mathscr{F}} h_{\mathbf{r}, \mathbf{j}} \mathbf{x}^{\mathbf{j}}, \quad \mathbf{r} \in \Delta \mathscr{F}\right\}
$$

называется полной системой $\mathscr{F}$-унитарных многочленов.

Лемма 6.1. Пусть $\mathscr{F} \subset \mathrm{N}_{0}^{k}-$ диаграмма Ферре $u$ идеал $I \triangleleft \mathscr{R}_{k}$ содержит некоторую полную систему (6.5) F्ञ-унитарных многочленов. Тогда множество $\mathfrak{X}$ вида (4.4) является системой образующих модуля ${ }_{R} T$.

Доказательство. Сначала заметим, что

$$
\boldsymbol{\omega}^{\mathbf{r}}=\sum_{\mathbf{j} \in \mathscr{F}} h_{\mathbf{r}, \mathbf{j}} \boldsymbol{\omega}^{\mathbf{j}}
$$

для каждого $\mathbf{r} \in \Delta \mathscr{F}$.

Зафиксируем некоторое допустимое упорядочение $\preceq$ множества $\mathbf{N}_{0}^{k}$. Индукцией по $\mathbf{j} \in \mathbf{N}_{0}^{k}$ относительно $\preceq$ покажем, что $\boldsymbol{\omega}^{\mathbf{i}}$ линейно выражается над $R$ через элементы множества $\mathfrak{A}$. Для $\mathbf{j} \in \mathscr{F} \cup \Delta \mathscr{F}$ утверждение очевидно. Пусть $\mathbf{j} \in \overline{\mathscr{F}} \backslash \Delta \mathscr{F}$ и для любого вектора $\mathbf{u} \in \mathscr{F}(\mathbf{j})\left(\right.$ см. (2.8)) элемент $\boldsymbol{\omega}^{\mathbf{u}}$ линейно выражается над $R$ через систему $\mathfrak{A}$. Рассмотрим элемент $\boldsymbol{\omega}^{\mathbf{j}}$. Так как $\mathbf{j}=\left(j_{1}, \ldots, j_{k}\right) \neq \mathbf{0}$, существует $s \in\{1, \ldots, k\}$ такое, что $j_{s}>0$. Тогда $\mathbf{j}-\mathbf{1}_{s} \in \mathscr{F}(\mathbf{j})$ и

$$
\boldsymbol{\omega}^{\mathbf{j}-\mathbf{1}_{s}}=\sum_{\mathbf{i} \in \mathscr{F}} h_{\mathbf{j}-\mathbf{1}_{s}, \mathbf{i}} \boldsymbol{\omega}^{\mathbf{i}}
$$

Значит,

$$
\boldsymbol{\omega}^{\mathbf{j}}=\sum_{\mathbf{i} \in \mathscr{F}} h_{\mathbf{j}-1_{s}, \mathbf{i}} \boldsymbol{\omega}^{\mathbf{i}+1_{s}}
$$

Заметим, что $\mathbf{i}+\mathbf{1}_{s} \in \mathscr{F} \cup \Delta \mathscr{F}$ для любого $\mathbf{i} \in \mathscr{F}$, и потому для каждого $\mathbf{i} \in \mathscr{F}$ элемент $\boldsymbol{\omega}^{\mathbf{i}+1_{s}}$ линейно выражается над $R$ через систему $\mathfrak{A}$. Теперь из последнего равенства следует, что и $\boldsymbol{\omega}^{\mathbf{j}}$ линейно выражается над $R$ через систему $\mathfrak{A}$. 
Каждой полной системе F्F-унитарных многочленов (6.5) соответствует система из $k$ эндоморфизмов $\Phi_{1}, \ldots, \Phi_{k}$ модуля $M^{|\mathscr{F}|}$, определяемых следующим образом: пусть $s \in\{1, \ldots, k\}$, тогда для любого набора $\delta[\mathscr{F}] \in M^{|\mathscr{F}|}$

$$
\Phi_{s}(\delta[\mathscr{F}])=\lambda_{s}[\mathscr{F}] \in M^{|\mathscr{F}|}
$$

где для любого вектора $\mathrm{i} \in \mathscr{F}$

$$
\lambda_{s}(\mathbf{i})= \begin{cases}\delta\left(\mathbf{i}+\mathbf{1}_{s}\right), & \text { если } \mathbf{i}+\mathbf{1}_{s} \in \mathscr{F}, \\ \sum_{\mathbf{j} \in \mathscr{F}} h_{\mathbf{r}, \mathbf{j}} \delta(\mathbf{j}), & \text { если } \mathbf{i}+\mathbf{1}_{s}=\mathbf{r} \in \Delta \mathscr{F} .\end{cases}
$$

Эти эндоморфизмы называются сопровождающими для системы многочленов $\Phi$. Пусть $\mathscr{E}=\operatorname{End}\left({ }_{R} M\right)$ - кольцо эндоморфизмов модуля ${ }_{R} M, \mathscr{E}_{k}=\mathscr{E}\left[x_{1}, \ldots, x_{k}\right]$.

Теорема 6.2 ([17], теоремы 2.1,2.2). Если F $\subset \mathbf{N}_{0}^{k}-$ диаграмма Ферре u $\Phi \subset \mathscr{R}_{k}-$ полная система $\mathscr{F}_{-}$-унитарных многочленов такая, что ее сопровождающие эндоморфизмы попарно перестановочны, то пара $\left\langle\mathscr{R}_{k} \Phi, \mathscr{F}\right\rangle$ образует $k$-ЛРC над модулем ${ }_{R} M$.

Если пара $\langle I, \mathscr{F}\rangle$ образует $k$-ЛРС над модулем ${ }_{R} M$, то существует полнал сис-

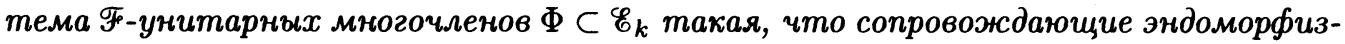
мъь $\Phi_{1}, \ldots, \Phi_{k}$ попарно перестановочны и пара $\left\langle\mathscr{E}_{k} \Phi, \mathscr{F}\right\rangle$ образует над модулем $\mathscr{E} M$ $k$-ЛРС, эквивалентный регистру $\langle I, \mathscr{F}\rangle$.

Теперь можно доказать следующее обращение теоремы 6.1, дающее необходимое условие того, что пара $\langle\dot{I}, \mathscr{F}\rangle$ образует регистр сдвига.

Теорема 6.3 ([19]). Пусть $I \triangleleft \mathscr{R}_{k}-$ унитарный идеал, $\mathscr{F} \subset \mathrm{N}_{0}^{k}-$ диаграмма Ферре и пара $\langle I, \mathscr{F}\rangle$ образует $k$-линейный регистр сдвига над квазифробениусовым модулем ${ }_{R} M$. Тогда факторколъцо $T$ естъ свободный $R$-модуль с базисом (4.4) и существует система $\Phi \subset \mathscr{R}_{k}$ F्न-унитарных многочленов тахая, что

$$
I=\mathscr{R}_{k} \Phi
$$

Доказательство. По теореме 6.2 существует регистр сдвига $\left\langle\mathscr{E}_{k} \Phi, \mathscr{F}\right\rangle$ над модулем є $M$, эквивалентный регистру $\langle I, \mathscr{F}\rangle$. Тогда для каждой $k$-последовательности $\mu \in L_{M}(I)$ справедливо равенство $\Phi \mu=0$. Поскольку ${ }_{R} M-$ квазифробениусов модуль, кольцо $\operatorname{End}\left({ }_{R} M\right)$ совпадает с кольцом гомотетий модуля ${ }_{R} M$ (это доказано, например, в [4]), то есть после естественного отождествления $\operatorname{End}\left({ }_{R} M\right)=R$. Это означает, что коэффициенты многочленов из системы $\Phi$, принадлежащие $\mathscr{E}$, в действительности суть скаляры из $R$, то есть

$$
\Phi=\left\{H_{\mathbf{i}}=\mathbf{x}^{\mathbf{i}}-\sum_{\mathbf{j} \in \mathscr{F}} h_{\mathbf{i}, \mathbf{j}} \mathbf{x}^{\mathbf{j}}, \mathbf{i} \in \Delta \mathscr{F}\right\} \subset \mathscr{R}_{k} .
$$

Значит, $\Phi$ принадлежит аннулятору $\operatorname{An}\left(L_{M}(I)\right)$ семейства $L_{M}(I)$ в кольце $\mathscr{R}_{k}$. Так как ${ }_{R} M$ - квазифробениусов модуль, справедливо равенство $\operatorname{An}\left(L_{M}(I)\right)=I$ (см. [4]). Таким образом, при сформулированных условиях $\Phi \subset I$. Теперь из леммы 6.1 следует, что $\mathfrak{U}$ - система образующих модуля ${ }_{R} T$.

Докажем, что $\mathfrak{U}$ - линейно независимая над $R$ система. Пусть

$$
\sum_{\mathbf{i} \in \mathscr{F}} h_{\mathbf{i}} \omega^{\mathbf{i}}=0
$$


то есть

$$
H=\sum_{\mathbf{i} \in \mathcal{F}^{*}} h_{\mathbf{i}} \mathbf{x}^{\mathbf{i}} \in I
$$

Зафиксируем произвольный вектор $\mathbf{r} \in \mathscr{F}$. Так как пара $\langle I, \mathscr{F}\rangle$ образует $k$-ЛРС, для каждого $a \in M$ существует единственная $k$-последовательность $\mu_{a} \in L_{M}(I)$ такая, что

$$
\forall \mathbf{j} \in \mathscr{F} \quad \mu_{a}(\mathbf{j})= \begin{cases}a, & \text { если } \mathbf{j}=\mathbf{r}, \\ 0, & \text { если } \mathbf{j} \neq \mathbf{r} .\end{cases}
$$

Рассмотрим последовательность $\nu=H \mu_{a}$. Так как $H \in I$, то $\nu=0$ и, в частности,

$$
\nu(\mathbf{0})=\sum_{\mathbf{i} \in \mathcal{F}} h_{\mathbf{i}} \mu_{a}(\mathbf{i})=h_{\mathbf{r}} a=0 .
$$

Итак, $h_{\mathbf{r}} a=0$ для каждого $a \in M$. Значит, $h_{\mathbf{r}} \in \operatorname{An}_{R}(M)=\{h \in R \mid h M=0\}$, то есть $h_{\mathbf{r}}$ принадлежит аннулятору модуля $M$ в кольце $R$. Квазифробениусов модуль ${ }_{R} M$ является точным, то есть $\operatorname{An}_{R}(M)=\{0\}$. Следовательно, $h_{\mathbf{r}}=0$. Значит, система $\mathfrak{U}$ свободна над $R$.

Докажем равенство (6.6). Положим $I_{1}=\mathscr{R}_{k} \Phi$. Включение $I_{1} \subseteq I$ следует из доказанного выше включения $\Phi \subset I$. Докажем обратное включение. Факторкольцо $S=\mathscr{R}_{k} / I_{1}$ можно представить в виде $\tilde{R}\left[\vartheta_{1}, \ldots, \vartheta_{k}\right]$, где $\tilde{R}=R / I \cap R, \vartheta_{s}=x_{s}+I_{1}$, $s=1, \ldots, k$. Так как по построению соответствующие системе $\Phi$ сопровождающие эндоморфизмы модуля $\check{C} M$ попарно перестановочны и являются также эндоморфизмами модуля ${ }_{R} M$, согласно теореме 6.2 пара $\left\langle I_{1}, \mathscr{F}\right\rangle$ образует $k$-ЛРС над модулем ${ }_{R} M$ и, как уже доказано, ${ }_{R} S$ есть свободный $R$-модуль с базисом $\mathfrak{B}=\left\{\boldsymbol{\vartheta}^{\mathbf{i}}, \mathbf{i} \in \mathscr{F}\right\}$. Рассмотрим отображение $\varphi: S \rightarrow T$ по правилу

$$
\forall A \in \mathscr{R}_{k} \quad \varphi(\tilde{A}(\boldsymbol{\vartheta}))=\tilde{A}(\boldsymbol{\omega}) .
$$

Ввиду условия $I_{1} \subseteq I$ это определение корректно, и $\varphi$ есть эпиморфизм свободных $R$-модулей с одинаковым числом образующих. Так как $R$ - артиново кольцо, то $\varphi$ - изоморфизм, то есть $\operatorname{Ker} \varphi=I / I_{1}=0$. Следовательно, $I_{1}=I$.

Следствие 6.1 ([19]). Пусть $I \triangleleft \mathscr{R}_{k}-$ унитарный идеал, F्F $\subset \mathbf{N}_{0}^{k}-$ диаграмма Ферре. Тогда пара $\langle I, \mathscr{F}\rangle$ образует $k$-линейный регистр сдвига над $Q F$-модулем ${ }_{R} M$, если и толъко если бакторколъцо $T=\mathscr{R}_{k} / I$ есть свободный $R$-модуль с базисом (4.4).

Возвращаясь к основной теме данной работы, мы получаем теперь следующие результаты. По-прежнему считаем, что на $\mathrm{N}_{0}^{k}$ зафиксировано некоторое допустимое упорядочение $\preceq$.

Теорема 6.4. Пусть $R$ - коммутативное артиново цепное колъцо, $I \triangleleft \mathscr{R}_{k}-$ унитарный идеал и $\chi$ - произвольная KCO идеала I (относителъно выбранного упорлдочения Қ) вида (4.11). Тогда справедливъ следующие утверждения.

(а) Если в (4.11) $t=0$, то есть $\chi_{0}$ - система Гребнера, порождающал идеал $I$, то пара $\left\langle I, \mathscr{F}_{0}\right\rangle$ образует $k$-линейный регистр сдвига над любым модулем ${ }_{R} M$.

(b) Если для некоторой диаграммы Ферре $\mathscr{F} \subset \mathbf{N}_{0}^{k}$ пара $\langle I, \mathscr{F}\rangle$ образует $k$-линейный регистр сдвига над колъцом $R$, то $t=0$ и $\left|\mathscr{F}_{\text {f }}\right|=\left|\mathscr{F}_{0}\right|$. 


\begin{tabular}{|l|l|l|}
\hline$(0,0)$ & $(1,0)$ & $(2,0)$ \\
\hline$(0,1)$ & \\
\cline { 1 - 1 } &
\end{tabular}

Pис. 1.

Доказательство. Утверждение (а) теоремы следует из предложения 4.4 и теоремы 6.1.

Для доказательства (b) заметим, что ${ }_{R} R-Q F$-модуль и по теореме 6.3 факторкольцо $T=\mathscr{R}_{k} / I$ есть свободный $R$-модуль ранга $|\mathscr{F}|$ с базисом $\mathfrak{A}=\left\{\boldsymbol{\omega}^{\mathbf{i}}, \mathbf{i} \in \mathscr{F}\right\}$, $\boldsymbol{\omega}^{\mathbf{i}}=\mathbf{x}^{\mathbf{i}}+I$. Теперь остается воспользоваться предложением 4.4.

Интересно, что утверждение (b) теоремы 6.4 нельзя дополнить утверждением о том, что идеал $I$ имеет такую $\mathrm{KCO}$ (относительно какого-либо упорядочения $\preceq$ ), для которой первоначальная диаграмма Ферре F्F является опорной. Другими словами, утверждение (а) теоремы не дает полного описания всех $k$-линейных регистров сдвига с характеристическим идеалом $I$.

Пример 6.1. Пусть $R=G F(2), k=2$. Рассмотрим диаграмму Ферре

$$
\mathscr{F}=\{(0,0),(0,1),(1,0),(0,2),(2,0)\},
$$

представленную на рис. 1, и полную систему $\mathscr{F}$-унитарных многочленов

$$
\begin{aligned}
\Phi=\left\{F_{(3,0)}=x_{1}^{3}+x_{1}^{2}, F_{(2,1)}\right. & =x_{1}^{2} x_{2}+x_{1}^{2}+x_{2}^{2}, \\
F_{(1,1)} & \left.=x_{1} x_{2}+x_{1}^{2}+x_{2}^{2}, F_{(1,2)}=x_{1} x_{2}^{2}+x_{2}^{2}, F_{(0,3)}=x_{2}^{3}+x_{1}^{2}\right\} .
\end{aligned}
$$

Положим $I=\mathscr{R}_{2} \Phi$. Тогда пара $\langle I, \mathscr{F}\rangle$ образует 2-ЛРС над полем $R$, причем не существует допустимого упорядочения множества $\mathrm{N}_{0}^{2}$ такого, что $\mathscr{F}_{F}=\mathscr{F}_{0}$.

Докажем утверждение примера 6.1. Заметим, что $I$ является унитарным идеалом, это легко следует из леммы 6.1 или того факта, что $I$ содержит унитарные многочлены

$$
F_{(3,0)}=x_{1}^{3}+x_{1}^{2}, \quad F_{(1,2)}+x_{2}\left(F_{(1,1)}+F_{(0,3)}\right)=x_{2}^{2}\left(x_{2}^{2}+x_{2}+1\right) .
$$

Докажем, что пара $\langle I, \mathscr{F}\rangle$ образует 2-ЛРС. Для этого, согласно теореме 6.2, достаточно показать перестановочность сопровождающих эндоморфизмов $\Phi_{1}$ и $\Phi_{2}$ модуля ${ }_{R} R^{|\mathscr{F}|}$, которые в нашем случае выглядят следующим образом:

$$
\begin{aligned}
& \Phi_{1}(\delta(0,0), \ldots, \delta(0,2))=(\delta(1,0), \delta(2,0), \delta(2,0), \delta(0,2)+\delta(2,0), \delta(0,2)), \\
& \Phi_{2}(\delta(0,0), \ldots, \delta(0,2))=(\delta(0,1), \delta(0,2)+\delta(2,0), \delta(0,2)+\delta(2,0), \delta(0 ; 2), \delta(2,0)),
\end{aligned}
$$


где $\delta(0,0), \ldots, \delta(0,2)$ - произвольные элементы поля $R$. Заметим, что

$$
\begin{aligned}
& \Phi_{1}\left(\Phi_{2}(\delta(0,0), \ldots, \delta(0,2))\right) \\
& =(\delta(0,2)+\delta(2,0), \delta(0,2)+\delta(2,0), \delta(0,2)+\delta(2,0), \delta(0,2), \delta(2,0)) \\
& \Phi_{2}\left(\Phi_{1}(\delta(0,0), \ldots, \delta(0,2))\right) \\
& =(\delta(0,2)+\delta(2,0), \delta(0,2)+\delta(2,0), \delta(0,2)+\delta(2,0), \delta(0,2), \delta(2,0))
\end{aligned}
$$

то есть эндоморфизмы $\Phi_{1}$ и $\Phi_{2}$, действительно, перестановочны, а значит, пара $\langle I, \mathscr{F}\rangle$ есть 2-ЛРС.

Допустим, что существует допустимое упорядочение $\preceq$ множества $\mathrm{N}_{0}^{2}$ такое, что $\mathscr{F}=\mathscr{F}_{0}$. Пусть $\psi-\mathrm{KCO}$ идеала $I$ относительно $\preceq$. Напомним, что над полем КСО унитарного идеала совпадает с соответствующим редуцированным базисом Гребнера, значит, $\psi=\bar{\psi}$ и $\mathscr{F}_{\psi}=\mathscr{F}_{F}=\mathscr{F}_{0}$. Так как

$$
\mathscr{K} \mathscr{F}=\{(3,0),(1,1),(0,3)\},
$$

условие $\mathscr{F}_{\psi}=\mathscr{F}$ равносильно тому, что $\psi=\left\{L_{1}, L_{2}, L_{3}\right\}$, где $\operatorname{ldg}\left(L_{1}\right)=(3,0)$, $\operatorname{ldg}\left(L_{2}\right)=(1,1)$ и $\operatorname{ldg}\left(L_{3}\right)=(0,3)$. Покажем, что $\psi=\left\{F_{(3,0)}, F_{(1,1)}, F_{(0,3)}\right\}$. Рacсмотрим многочлен $H_{1}=L_{1}-F_{(3,0)}$. Выполняется включение $\subseteq\left(H_{1}\right) \subseteq \mathscr{F}$, то есть $H_{1} \in N(\psi)$. Так как $H_{1} \in I$, отсюда следует, что $H_{1}=0$ и $L_{1}=F_{(3,0)}$. Два оставшихся равенства доказываются аналогично. Далее покажем, что $\operatorname{ldg}\left(F_{(1,1)}\right) \neq(1,1)$. Так как $\preceq$ - отношение линейного порядка на $\mathbf{N}_{0}^{2}$, то либо $(1,0) \prec(0,1)$, либо $(0,1) \prec(1,0)$. Допустим, что $(1,0) \prec(0,1)$. Тогда $(1,1) \prec(0,2)$ и $\operatorname{ldg}\left(F_{(1,1)}\right)=(0,2)$. Случай $(0,1) \prec(1,0)$ рассматривается аналогично. Значит, система $\psi$ не является КСО (редуцированным базисом Гребнера) идеала $I$ относительно упорядочения $\preceq$. Утверждение примера доказано.

Построим 2-ЛРС $\left\langle I, \mathscr{F}_{0}\right\rangle$ эквивалентный регистру $\langle I, \mathscr{F}\rangle$ из примера 6.1 такой, что $\chi_{0}$ - система Гребнера.

Пример 6.2. При тех же условиях, что и в предыдущем примере, рассмотрим диаграмму Ферре

$$
\mathscr{F}_{0}=\{(0,0),(0,1),(1,0),(0,2),(1,1)\},
$$

представленную на рис. 2 , и полную систему $\mathscr{F}_{0}$-унитарных многочленов

$$
\begin{aligned}
\Phi_{0}=\left\{G_{(3,0)}=x_{1}^{3}+x_{1}^{2}, G_{(2,1)}\right. & =x_{1}^{2} x_{2}+x_{1} x_{2}, \\
& \left.G_{(1,2)}=x_{1} x_{2}^{2}+x_{1}^{2}+x_{1} x_{2}, G_{(0,2)}=x_{2}^{2}+x_{1}^{2}+x_{1} x_{2}\right\} .
\end{aligned}
$$

Положим $I_{0}=\mathscr{R}_{2} \Phi_{0}$. Покажем, что $I_{0}=I$ и пара $\left\langle I, \mathscr{F}_{0}\right\rangle$ есть 2-ЛРС над $R$, эквивалентный регистру $\langle I, \mathscr{F}\rangle$ из примера 6.1 , причем множество

$$
\chi_{0}=\left\{G_{(3,0)}, G_{(2,1)}, G_{(0,2)}\right\}
$$

есть КСО идеала $I$ при чисто лексикографическом $\left(x_{1} \prec x_{2}\right)$ упорядочении множества $\mathbf{N}_{0}^{2}$.

Докажем утверждения примера 6.2.

Так как

$$
G_{(1,2)}=x_{1} G_{(0,2)}+G_{(3,0)}+G_{(2,1)},
$$




\begin{tabular}{|l|l|l|}
\hline$(0,0)$ & $(1,0)$ & $(2,0)$ \\
\hline$(0,1)$ & $(2,2)$ & \\
\end{tabular}

\section{Puc. 2.}

идеал $I_{0}$ порождается системой многочленов $\chi_{0}$. Легко заметить, что

$$
\begin{aligned}
& G_{(3,0)}=F_{(3,0)}, \quad G_{(2,1)}=F_{(2,1)}+F_{(1,1)}, \quad F_{(2,1)}=G_{(2,1)}+G_{(0,2)}, \\
& F_{(1,1)}=G_{(0,2)}, \quad F_{(1,2)}=G_{(1,2)}+G_{(0,2)}, \quad F_{(0,3)}=x_{2} G_{(0,2)}+G_{(2,1)}+G_{(1,2)} \text {, }
\end{aligned}
$$

поэтому, $I_{0}=I$.

Докажем, что $\chi_{0}$ есть КСО идеала $I$. Для этого достаточно показать, что множество $\chi_{0}$ есть редуцированный базис Гребнера идеала $I$. Воспользуемся аппаратом $S$-полиномов. Напомним, что конечное подмножество $\varphi \subset \mathscr{P}_{k}$ есть базис Гребнера идеала $J=\mathscr{P}_{k} \varphi$ тогда и только тогда, когда для любых двух различных многочленов $H, F \in \varphi S$-полином $S P(H, F)$ либо равен 0 , либо редуцируется к 0 по модулю множества $\varphi$ (см., например, [2]).

Покажем редуцируемость к 0 по модулю множества $\chi_{0}$ всех трех возможных $S$-полиномов:

$$
\begin{aligned}
S P\left(G_{(3,0)}, G_{(2,1)}\right) & =x_{1}^{3} x_{2}+x_{1}^{2} x_{2}+x_{1}^{3} x_{2}+x_{1}^{2} x_{2}=0 \\
S P\left(G_{(3,0)}, G_{(0,2)}\right) & =x_{1}^{3} x_{2}^{2}+x_{1}^{2} x_{2}^{2}+x_{1}^{3} x_{2}^{2}+x_{1}^{5}+x_{1}^{4} x_{2} \\
& =x_{2}\left(G_{(2,1)}+x_{1} x_{2}\right)+x_{1}^{5}+x_{1}^{4} x_{2} \\
& =x_{2} G_{(2,1)}+x_{1} G_{(0,2)}+x_{1}^{3}+x_{1}^{2} x_{2}+x_{1}^{5}+x_{1}^{4} x_{2} \\
& =x_{2} G_{(2,1)}+x_{1} G_{(0,2)}+x_{1}^{2} G_{(2,1)}+x_{1}^{3} x_{2}+x_{1}^{3}+x_{1}^{2} x_{2}+x_{1}^{5} \\
& =x_{2} G_{(2,1)}+x_{1} G_{(0,2)}+x_{1}^{2} G_{(2,1)}+x_{1} G_{(2,1)}+x_{1}^{5}+x_{1}^{3} \\
& =x_{2} G_{(2,1)}+x_{1} G_{(0,2)}+x_{1}^{2} G_{(2,1)}+x_{1} G_{(2,1)}+\left(x_{1}^{2}+x_{1}\right) G_{(3,0)} \\
S P\left(G_{(2,1)}, G_{(0,2)}\right) & =x_{1}^{2} x_{2}^{2}+x_{1} x_{2}^{2}+x_{1}^{2} x_{2}^{2}+x_{1}^{4}+x_{1}^{3} x_{2} \\
& =x_{1} G_{(0,2)}+x_{1}^{3}+x_{1}^{2} x_{2}^{2}+x_{1}^{4}+x_{1}^{3} x_{2} \\
& =x_{1} G_{(0,2)}+x_{1} G_{(2,1)}+x_{1}^{3}+x_{1}^{4}=x_{1} G_{(0,2)}+x_{1} G_{(2,1)}+x_{1} G_{(3,0)} .
\end{aligned}
$$

Итак, все $S$-полиномы редуцируются к 0 по модулю множества $\chi_{0}$, значит, $\chi_{0}$ есть КСО идеала $I$. Тогда, согласно теореме 6.4 , пара $\left\langle I, \mathscr{F}_{0}\right\rangle$ есть 2 -ЛРС.

\section{7. Система образующих $k$-ЛРП семейства с заданным характеристическим унитарным идеалом}

Пусть по-прежнему $R$ - коммутативное артиново цепное кольцо и $I \triangleleft \mathscr{R}_{k}-$ собственный унитарный идеал. Зафиксируем некоторое допустимое упорядочение $\preceq$ мно- 
жества $\mathrm{N}_{0}^{k}$ и построим систему образующих ЛРП-семейства $L_{R}(I)$, имея $\mathrm{KCO}(4.11)$ идеала $I$.

Сначала рассмотрим случай, когда в (4.11) $t=0$, то есть, согласно предложению $4.4, \mathrm{KCO}$ идеала $I$ есть $\chi=\chi_{0}$ - система Гребнера с опорной диаграммой $\mathscr{F}_{F}=\mathscr{F}_{0}$. Заметим, что частный случай этой ситуации - все ЛРП-семейства над полем. По теореме 6.4 при сформулированных условиях пара $\langle I, \mathscr{F}\rangle$ образует $k$-ЛРС, то есть для любого вектора $\mathrm{c} \in R^{|\mathscr{F}|}$ существует единственная $k$-последовательность $\mu \in L_{R}(I)$ с начальным заполнением $\mu[\mathscr{F}]=\mathbf{c}$. Для каждого $\mathbf{i} \in \mathscr{F}$ через $e_{\chi}^{\mathbf{i}}$ обозначим $k$-последовательность из $L_{R}(I)$ с начальным заполнением $e_{\chi}^{\mathbf{i}}[\mathscr{F}]$, определяемым условием

$$
\forall \mathbf{j} \in \mathscr{F} \quad e_{\chi}^{\mathbf{i}}(\mathbf{j})= \begin{cases}e, & \text { если } \mathbf{j}=\mathbf{i} \\ 0, & \text { если } \mathbf{j} \neq \mathbf{i}\end{cases}
$$

Очевидно, что при сделанном предположении $L_{R}(I)$ есть свободный $R$-модуль с базисом $\left\{e_{\chi}^{\mathbf{i}}, \mathbf{i} \in \mathscr{F}\right\}$.

Назовем множеством внутренних углов диаграммы Ферре $\mathscr{F}_{F}$ множество $\mathscr{C} \mathscr{F}$ всех максимальных элементов частично упорядоченного множества $(\mathscr{F}, \leqslant)$.

Теорема 7.1. Если $\langle I, \mathscr{F}\rangle$ есть $k$-ЛPC над кольцом $R$, то множество $E_{\chi}=\left\{e_{\chi}^{\mathrm{i}} \mid \mathbf{i} \in \mathscr{C F}\right\}$ есть система образующих $\mathscr{R}_{k}$-модуля $L_{R}(I)$.

Доказательство. Для каждого $\mathbf{u} \in \mathscr{F}$ выберем и зафиксируем вектор $\mathbf{i} \in \mathscr{C F}$ со свойством $\mathbf{u} \leqslant \mathbf{i}$ и положим

$$
a^{\mathbf{u}}=\mathbf{x}^{1} e_{\chi}^{\mathbf{i}}, \quad \mathbf{l}=\mathbf{i}-\mathbf{u}
$$

Для доказательства теоремы достаточно показать, что система последовательностей

$$
\left\{a^{\mathbf{u}} \mid \mathbf{u} \in \mathscr{F}\right\}
$$

очевидно, принадлежащая семейству $L_{R}(I)$, порождает его как $R$-модуль. Нам потребуются две леммы.

Лемма 7.1. Если $R-$ поле, то есть $n=1$, то для любы $\mathbf{u} \in \mathscr{F}, \mathbf{j} \in \mathbf{N}_{0}^{k}$ справедливы соотношения

$$
a^{\mathbf{u}}(\mathbf{u})=e, \quad\left(a^{\mathbf{u}}(\mathbf{j}) \neq 0\right) \Rightarrow(\mathbf{u} \preceq \mathbf{j})
$$

Доказателъство. Покажем сначала, что

$$
\forall \mathbf{s} \in \mathbf{N}_{0}^{k} \quad\left(e_{\chi}^{\mathbf{i}}(\mathbf{s}) \neq 0\right) \Rightarrow(\mathbf{i} \preceq \mathbf{s}) .
$$

Если $\mathbf{s} \in \mathscr{F}$, то из условия $e_{\chi}^{\mathbf{i}}(\mathbf{s}) \neq 0$ и определения последовательности $e_{\chi}^{\mathbf{i}}$ следует, что $\mathbf{s}=\mathbf{i}$ и $\mathbf{i} \preceq \mathbf{s}$.

Пусть теперь $\mathbf{s} \in \overline{\mathscr{F}}$. Воспользуемся соотношением $e_{\chi}^{\mathbf{i}}(\mathbf{s})=\left(\mathbf{x}^{\mathbf{s}} e_{\chi}^{\mathbf{i}}\right)(\mathbf{0})$. Используя терминологию, обозначения и результаты леммы 2.1 , можно сказать, что $\mathbf{s}-$ ординарный показатель, и так как многочлен (2.12) принадлежит идеалу $I$, то

$$
e_{\chi}^{\mathbf{i}}(\mathbf{s})=\sum_{\mathbf{j} \in \mathcal{F} \cap \mathscr{F}(\mathbf{s})} g_{\mathbf{s} \mathbf{j}} e_{\chi}^{\mathbf{i}}(\mathbf{j}) .
$$


Отсюда, по определению последовательности $e_{\chi}^{\mathbf{i}}$, получаем, что $e_{\chi}^{\mathbf{i}}(\mathbf{s})=g_{\mathbf{s i}} \neq 0$, и потому $\mathbf{i} \in \mathscr{F}_{(\mathbf{s}}(\mathbf{s})$, то есть $(\mathbf{i} \preceq \mathbf{s})$.

Теперь из (7.2) получаем, что $a^{\mathbf{u}}(\mathbf{j})=e_{\chi}^{\mathbf{i}}(\mathbf{l}+\mathbf{j})$ и ввиду $(7.4)$

$$
\left(a^{\mathbf{u}}(\mathbf{j}) \neq 0\right) \Rightarrow(\mathbf{i} \preceq \mathbf{l}+\mathbf{j}) \Rightarrow(\mathbf{u} \preceq \mathbf{j}) .
$$

Лемма 7.2. Если $R$ - поле, то система ЛРП (7.3) есть базис пространства $L_{R}(I)$.

Доказательство. Так как размерность пространства $L_{R}(I)$ равна числу $|\mathscr{F}|$ элементов системы (7.3), достаточно показать, что любая ЛРП $\mu \in L_{R}(I)$ линейно выражается через эту систему.

Будем считать, что элементы диаграммы $\mathscr{F}=\left\{\mathbf{i}_{1}, \ldots, \mathbf{i}_{d}\right\}$ перенумерованы в соответствии с упорядочением ঝ:

$$
\mathbf{i}_{1} \prec \mathbf{i}_{2} \prec \ldots \prec \mathbf{i}_{d} .
$$

Построим систему последовательностей $\mu_{s}, s=0,1, \ldots, d$, рекурсивно по закону

$$
\mu_{0}=\mu, \quad \mu_{s}=\mu_{s-1}-\mu_{s-1}\left(\mathbf{i}_{s}\right) a^{\mathbf{i}_{s}}, \quad s=1, \ldots, d .
$$

Очевидно, что $\mu_{s} \in L_{R}(I)$. Индукцией по $s$ с использованием леммы 7.1 нетрудно доказать, что $\mu_{s}\left(\mathbf{i}_{t}\right)=0$ для $t=1, \ldots, s$, и потому $\mu_{d}[\mathscr{F}]=0$. Так как любая ЛРП из $L_{R}(I)$ однозначно определяется диаграммой значений на $\mathscr{F}$, последнее равенство означает, что $\mu_{d}=0$. Следовательно,

$$
\mu=\sum_{s=1}^{d} \mu_{s-1}\left(\mathbf{i}_{s}\right) a^{\mathbf{i}_{*}} .
$$

Рассмотрим теперь общий случай, когда $n \geqslant 1$. Зададим гомоморфизм $R$-модулей $\varphi: L_{R}(I) \rightarrow L_{\bar{R}}(\bar{I})$ равенством $\varphi(\mu)=\bar{\mu}$. Гомоморфизм $\varphi$ есть эпиморфизм, так как семейство $L_{\bar{R}}(\bar{I})$ есть, очевидно, пространство над $\bar{R}$ с базисом, состоящим из ЛРП

$$
\bar{e}_{\bar{\chi}}^{\mathbf{i}}=\varphi\left(e_{\chi}^{\mathbf{i}}\right), \quad \mathbf{i} \in \mathscr{F} .
$$

Кроме того, справедливо равенство $\operatorname{Ker} \varphi=\pi L_{R}(I)$. Действительно, очевидно, что $\operatorname{Ker} \varphi=L_{R}(I) \cap \pi R^{\langle k\rangle}$. Любая последовательность $\mu \in L_{R}(I) \cap \pi R^{\langle k\rangle}$ представляется в виде $\mu=\pi \nu$, где $\nu \in R^{\langle k\rangle}$. При сформулированных условиях на идеал $I$, последовательность $\nu$ в этом представлении можно выбрать из $L_{R}(I)$. Действительно, выберем $\mathbf{c} \in R^{d}$ так, что $\mu[\mathscr{F}]=\pi \mathbf{c}$. Так как пара $\langle I, \mathscr{F}\rangle$ есть ЛРС, существует ЛРП $\nu \in L_{R}(I)$ такая, что $\nu[\mathscr{F}]=$ c. Тогда $\pi \nu \in L_{R}(I),(\pi \nu)[\mathscr{F}]=\mu[\mathscr{F}]$ и, следовательно, $\pi \nu=\mu$.

Так как $\operatorname{Ker} \varphi=(\pi R) L_{R}(I)$ и $\pi R$ - нильпотентный идеал, по лемме Накаямы система последовательностей (7.3) порождает $R$-модуль $L_{R}(I)$, поскольку по лемме 7.2 система последовательностей $\left\{\overline{\mathbf{a}}^{\mathbf{u}}, \mathbf{u} \in \mathscr{F}\right\}$ порождает $\bar{R}$-модуль $L_{\bar{R}}(\bar{I})$. Теорема 7.1 доказана.

Для описания системы образующих семейства $L_{R}(I)$ в случае, когда параметр $t$ идеала $I$ больше 0 , нам потребуется несколько дополнительных понятий и утверждений. 
Пусть $\mathscr{F} \subset \mathbf{N}_{0}^{k}-$ непустая диаграмма Ферре и

$$
\psi=\left\{G_{\mathbf{u}}=\mathbf{x}^{\mathbf{u}}-\sum_{\mathbf{j} \in \mathscr{F}^{\prime}} g_{\mathbf{u}, \mathbf{j}} \mathbf{x}^{\mathbf{j}}, \mathbf{u} \in \mathscr{K} \mathscr{F}\right\} \subset \mathscr{R}_{k}
$$

- круллева система многочленов с опорной диаграммой $\mathscr{F}$. Для каждого $\mathbf{u} \in \mathbf{N}_{0}^{k}$ зафиксируем некоторую нормальную форму

$$
H_{\mathbf{u}}(\mathbf{x})=\sum_{\mathbf{j} \in \mathscr{F}_{F}} h_{\mathbf{u}, \mathbf{j}} \mathbf{x}^{\mathbf{j}}
$$

монома $\mathbf{x}^{\mathbf{u}}$ по модулю множества $\psi$. Заметим, что для каждого $\mathbf{u} \in F_{F}$ моном $\mathbf{x}^{\mathbf{u}}$ является нормальным по модулю $\psi$ и $N F\left(\mathbf{x}^{\mathbf{u}}, \psi\right)=\left\{\mathbf{x}^{\mathbf{u}}\right\}$, то есть $H_{\mathbf{u}}(\mathbf{x})=\mathbf{x}^{\mathbf{u}}$. Так же однозначно определены нормальные формы мономов $\mathbf{x}^{\mathbf{u}}, \mathbf{u} \in \mathscr{K} \mathscr{F}$, поскольку для каждого $\mathbf{u} \in \mathscr{K} \mathscr{F}$ имеет место равенство

$$
N F\left(\mathbf{x}^{\mathbf{u}}, \psi\right)=\left\{\mathbf{x}^{\mathbf{u}}-G_{\mathbf{u}}=\sum_{\mathbf{j} \in \mathscr{F}} g_{\mathbf{u}, \mathbf{j}} \mathbf{x}^{\mathbf{j}}\right\}
$$

и потому

$$
H_{\mathbf{u}}=\sum_{\mathbf{j} \in \mathscr{F}^{F}} g_{\mathbf{u}, \mathbf{j}} \mathbf{x}^{\mathbf{j}}
$$

Разверткой отрезка $\mu[\mathscr{F}] \in R^{|\mathscr{F}|}$ с помощью множества $\psi$ назовем $k$-последовательность $\nu \in R^{\langle k\rangle}$ вида

$$
\nu(\mathbf{u})=\sum_{\mathbf{j} \in \mathscr{F}} h_{\mathbf{u}, \mathbf{j}} \mu(\mathbf{j}), \quad \mathbf{u} \in \mathbf{N}_{\mathbf{0}}^{k}
$$

Заметим, что при этом $\nu[\mathscr{F}]=\mu[\mathscr{F}]$, так как для каждого $\mathbf{u} \in \mathscr{F}$, выполняется равенство $H_{\mathbf{u}}(\mathbf{x})=\mathbf{x}^{\mathbf{u}}$.

Лемма 7.3. Пусть $\mathscr{F} \subset \mathbf{N}_{0}^{k}-$ непустая диаграмма Ферре, $\mu, \nu \in R^{\langle k\rangle}$ u последовательность $\nu$ является разверткой отрезка $\mu[\mathscr{F}]$ с помощъю круллевой системъ $\psi$ F्न-унитарных многочленов. Тогда для любых $G \in \psi u \mathbf{i} \in \mathbf{N}_{0}^{k}$ существует многочлен $H \in J=\mathscr{R}_{k} \psi$ maxoü, чmo $\subseteq(H) \subseteq \mathscr{F} u(G \nu)(\mathbf{i})=(H \mu)(\mathbf{0})$.

Доказательство. Пусть $\operatorname{ldg}(G)=\mathbf{u}$. Тогда

$$
\begin{aligned}
G & =\mathbf{x}^{\mathbf{u}}-\sum_{\mathbf{j} \in \mathcal{F}} g_{\mathbf{u}, \mathbf{j}} \mathbf{x}^{\mathbf{j}}, \\
\mathbf{x}^{\mathbf{i}} G & =\mathbf{x}^{\mathbf{i}+\mathbf{u}}-\sum_{\mathbf{j} \in \mathcal{F}_{F}} g_{\mathbf{u}, \mathbf{j}} \mathbf{x}^{\mathbf{i}+\mathbf{j}} \in J .
\end{aligned}
$$

Так как

$$
\mathbf{x}^{\mathbf{i}+\mathbf{j}}-H_{\mathbf{i}+\mathbf{j}}=\mathbf{x}^{\mathbf{i}+\mathbf{j}}-\sum_{\mathbf{i} \in \mathcal{F}} h_{\mathbf{i}+\mathbf{j}, 1} \mathbf{x}^{\mathbf{l}} \in J
$$

для любого $\mathbf{j} \in \mathscr{F}$, многочлен

$$
H(\mathbf{x})=\mathbf{x}^{\mathbf{i}} G-\left(\mathbf{x}^{\mathbf{i}+\mathbf{u}}-H_{\mathbf{i}+\mathbf{u}}\right)+\sum_{\mathbf{j} \in \mathscr{F}^{\prime}} g_{\mathbf{u}, \mathbf{j}}\left(\mathbf{x}^{\mathbf{i}+\mathbf{j}}-H_{\mathbf{i}+\mathbf{j}}\right)
$$


принадлежит $J$, то есть

$$
H(\mathbf{x})=\sum_{\mathbf{l} \in \mathscr{F}} h_{\mathbf{i}+\mathbf{u}, \mathbf{l}} \mathbf{x}^{\mathbf{1}}-\sum_{\mathbf{j} \in \mathscr{F}} g_{\mathbf{u}, \mathbf{j}}\left(\sum_{\mathbf{l} \in \mathscr{F}^{\mathscr{F}}} h_{\mathbf{i}+\mathbf{j}, \mathbf{1}} \mathbf{x}^{\mathbf{1}}\right) \in J .
$$

Очевидно, что $\subseteq(H) \subseteq \mathscr{F}$. Кроме того, из определения последовательности $\nu$ и (7.7), следует цепочка равенств

$$
\begin{aligned}
(G \nu)(\mathbf{i}) & =\nu(\mathbf{i}+\mathbf{u})-\sum_{\mathbf{j} \in \mathscr{F}} h_{\mathbf{u}, \mathbf{j}} \nu(\mathbf{i}+\mathbf{j}) \\
& =\sum_{\mathbf{l} \in \mathscr{F}} h_{\mathbf{i}+\mathbf{u}, \mathbf{1}} \mu(\mathbf{l})-\sum_{\mathbf{j} \in \mathscr{F}} h_{\mathbf{u}, \mathbf{j}}\left(\sum_{\mathbf{l} \in \mathscr{F}} h_{\mathbf{i}+\mathbf{j}, \mathbf{l}} \mu(\mathbf{l})\right)=(H \mu)(\mathbf{0})
\end{aligned}
$$

Лемма доказана.

Пусть унитарный идеал $I \triangleleft \mathscr{R}_{k}$ имеет $\mathrm{KCO} \chi$ вида

$$
\chi=\chi_{0} \cup \pi^{a_{1}} \chi_{1} \cup \ldots \cup \pi^{a_{t}} \chi_{t} .
$$

Напомним, что $J_{i}=\left(I: \pi^{a_{i}}\right)$ для $i=0,1, \ldots, t$. Положим $J_{t+1}=\mathscr{R}_{k}$. Согласно (C4), $J_{i}$ есть унитарный идеал с главной частью $\mathrm{KCO} \chi_{i}$, для $i=0,1, \ldots, t$. КСО идеала $J_{t+1}$ есть $\chi_{t+1}=\{e\}$.

Лемма 7.4. Пусть $r \in\{0,1, \ldots, t\}, H \in \mathscr{R}_{k}$. Тогда из условий $H \in J_{r}, \Im(H) \subseteq \mathscr{F}_{r}$ cледyem, что $H=\pi^{a_{r+1}-a_{r}} H_{1}$, где $H_{1} \in J_{r+1}$.

Доказательство. Пусть $H \in J_{r}, \Im(H) \subseteq \mathscr{F}_{r}$. Тогда $\pi^{a_{r}} H \in I$ и

$$
\Im\left(\pi^{a_{r}} H\right) \subseteq \Im(H) \subseteq \mathscr{F}_{r} .
$$

Отсюда и из (C1) следует, что $\left\|\pi^{a_{r}} H\right\| \geqslant a_{r+1}$, то есть $H=\pi^{a_{r+1}-a_{r}} H_{1}$, где $H_{1} \in \mathscr{R}_{k}$. Далее, так как $\pi^{a_{r}} H=\pi^{a_{r+1}} H_{1} \in I$, то $H_{1} \in J_{r+1}$.

Лемма 7.5. Пустъ $\nu \in R^{\langle k\rangle}$ - развертка отрезка $\nu\left[\mathscr{F}_{r}\right]$ с помощъю круллевой системъ $\chi_{r} \neq\{e\}, r=0,1, \ldots, t$. Тогда для любого $F \in J_{r}$ справедливо соотношение

$$
F \nu \equiv 0 \quad\left(\bmod \pi^{a_{r+1}-a_{r}}\right)
$$

Доказателъство. Согласно лемме 7.3 для любых $G \in \chi_{r}, \mathbf{u} \in \mathbf{N}_{0}^{k}$ существует многочлен $H \in J_{r}$ такой, что $(G \nu)(\mathbf{u})=(H \nu)(\mathbf{0})$ и $\subseteq(H) \subseteq \mathscr{F}_{r}$. Тогда по лемме 7.4 $H=\pi^{a_{r+1}-a_{r}} H_{1}$, где $H_{1} \in J_{r+1}$. Значит, $(G \nu)(\mathbf{u})=\pi^{a_{r+1}-a_{r}}\left(H_{1} \nu\right)(\mathbf{0})$. Так как вектор $\mathbf{u}$ выбран произвольно, отсюда следует, что $G \nu \equiv 0\left(\bmod \pi^{a_{r+1}-a_{r}}\right)$. Пусть теперь $F \in J_{r}$. Из (C4) следует, что $F=K+\pi^{a_{r+1}-a_{r}} L$, где $K \in \mathscr{R}_{k} \chi_{r}, L \in J_{r+1}$. Значит, $F \nu \equiv 0\left(\bmod \pi^{a_{r+1}-a_{r}}\right)$.

Предложение 7.1. Для любого $s=0,1, \ldots, t u$ любого $\mu \in L_{\bar{R}}\left(\bar{J}_{s}\right)$ существует $k$-последовательность $\omega \in R^{\langle k\rangle}$, удовлетворяющая условию

$$
\pi^{n-a_{s+1}} \omega \in L_{R}(I), \quad \bar{\omega}=\mu .
$$


Доказателъство. Рассмотрим сначала случай $s=t, \chi_{t}=\{e\}$. Заметим, что при этом $\bar{\chi}_{t}=\{e\}, \bar{J}_{t}=\overline{\mathscr{R}}_{k}$ и $\mu=0$. Тогда $k$-последовательность $\omega=0$ удовлетворяет условиям (7.9). Пусть далее $s<t$ или $s=t$ и $\chi_{t} \neq\{e\}$. Отметим, что для любой последовательности $\omega \in R^{\langle k\rangle}$ условие $\pi^{n-a_{s+1}} \omega \in L_{R}(I)$ равносильно тому, что для любого $i=0,1, \ldots, s$ и любого $F \in J_{s-i}$ выполняется равенство $\pi^{n-a_{s+1}+a_{s-i}} F \omega=0$, то есть равносильно условию

$$
\forall i \in\{0,1, \ldots, s\} \forall F \in J_{s-i} \quad F \omega \equiv 0 \quad\left(\bmod \pi^{a_{s+1}-a_{s-i}}\right) .
$$

Построим $s+2$ последовательности $\mu_{0}, \mu_{1}, \ldots, \mu_{s+1}$ рекурсивно, так что $\mu_{0} \in R^{\langle k\rangle}$, $\bar{\mu}_{0}=\mu$ и последовательность $\mu_{i+1}$ есть развертка отрезка $\mu_{i}\left[\mathscr{F}_{s-i}\right]$ с помощью множества $\chi_{s-i}, i=0,1, \ldots, s$. Покажем, что тогда $\boldsymbol{\omega}=\mu_{s+1}-$ искомая последовательность.

Докажем равенство $\bar{\mu}_{1}=\mu$. По построению для любого $\mathbf{u} \in \mathbf{N}_{0}^{k}$ справедливо равенство

$$
\mu_{1}(\mathbf{u})=\sum_{\mathbf{j} \in \mathscr{F}_{s}} h_{\mathbf{u}, \mathbf{j}} \mu_{0}(\mathbf{j}),
$$

где

$$
\sum_{\mathbf{j} \in \mathscr{F}_{s}} h_{\mathbf{u}, \mathbf{j}} \mathbf{x}^{\mathbf{j}} \in N F\left(\mathbf{x}^{\mathbf{u}}, \chi_{s}\right) .
$$

Так как

$$
F=\mathbf{x}^{\mathbf{u}}-\sum_{\mathbf{j} \in \mathscr{F}_{s}} h_{\mathbf{u}, \mathbf{j}} \mathbf{x}^{\mathbf{j}} \in J_{s}
$$

из условий $\mu=\bar{\mu}_{0}$ и $\mu \in L_{\bar{R}}\left(\bar{J}_{s}\right)$ следует сравнение $F \mu_{0} \equiv 0(\bmod \pi)$. Значит,

$$
\mu_{0}(\mathbf{u}) \equiv \sum_{\mathbf{j} \in \mathscr{F}_{s}} h_{\mathbf{u}, \mathbf{j}} \mu_{0}(\mathbf{j}) \quad(\bmod \pi)
$$

Следовательно, $\mu_{0}(\mathbf{u}) \equiv \mu_{1}(\mathbf{u})(\bmod \pi)$. Так как вектор и выбран произвольно, отсюда следуют сравнение $\mu_{1} \equiv \mu_{0}(\bmod \pi)$ и нужное равенство $\bar{\mu}_{1}=\mu$.

Заметим, что если $s=0$, то $\boldsymbol{\omega}=\mu_{1}$ и (7.10) следует из леммы 7.5 при $r=0$, так как последовательность $\mu_{1}=\omega$ является разверткой отрезка $\mu_{0}\left[\mathscr{F}_{0}\right]$ с помощью множества $\chi_{0}$.

Пусть далее $s>0$. Индукцией по $i=0,1, \ldots, s$ докажем, что последовательности $\mu_{1}, \ldots, \mu_{s+1}$ удовлетворяют условиям

$$
\begin{aligned}
& \mu_{i+1} \equiv \mu_{i} \quad\left(\bmod \pi^{a_{s+1}-a_{s-i+1}}\right), \quad i=0,1, \ldots, s, \\
& \forall F \in J_{s-i} \quad F \mu_{i+1} \equiv 0 \quad\left(\bmod \pi^{a_{s+1}-a_{s-i}}\right), \quad i=0,1, \ldots, s .
\end{aligned}
$$

Пусть $i=0$. Пункт (7.11) в данном случае тривиален. При $i=0$ условие (7.12) имеет вид

$$
\forall F \in J_{s} \quad F \mu_{1} \equiv 0 \quad\left(\bmod \pi^{a_{s+1}-a_{s}}\right),
$$

и по лемме 7.5 оно выполняется, так как по условию последовательность $\mu_{1}$ является разверткой отрезка $\mu_{0}\left[\mathscr{F}_{s}\right]=\mu_{1}\left[\mathscr{F}_{s}\right]$ с помощью множества $\chi_{s}$.

Допустим, что условия (7.11) и (7.12) выполняются для всех $i \in\{0,1, \ldots, d-1\}$, $d \leqslant s$. Докажем, что они верны для $i=d$. По построению, для любого $\mathbf{u} \in \mathbf{N}_{0}^{k}$ выполняется равенство

$$
\mu_{d+1}(\mathbf{u})=\sum_{\mathbf{j} \in \mathscr{F}_{s-d}} h_{\mathbf{u}, \mathbf{j}} \mu_{d}(\mathbf{j})
$$


где

$$
\sum_{\mathbf{j} \in \mathscr{F}_{s-d}} h_{\mathbf{u}, \mathbf{j}} \mathbf{x}^{\mathbf{j}} \in N F\left(\mathbf{x}^{\mathbf{u}}, \chi_{s-d}\right)
$$

Так как

$$
F=\mathbf{x}^{\mathbf{u}}-\sum_{\mathbf{j} \in \mathscr{F}_{s-d}} h_{\mathbf{u}, \mathbf{j}} \mathbf{x}^{\mathbf{j}} \in J_{s-d} \subset J_{s-d+1}
$$

по уже доказанному $F \mu_{d} \equiv 0\left(\bmod \pi^{a_{s+1}-a_{s-d+1}}\right)$ и верно (7.11).

Докажем (7.12) для $i=d$. Так как последовательность $\mu_{d+1}$ является разверткой отрезка $\mu_{d}\left[\mathscr{F}_{s-d}\right]=\mu_{d+1}\left[\mathscr{F}_{s-d}\right]$ с помощью множества $\chi_{s-d}$, согласно лемме 7.3 для любых $G \in \chi_{s-d}, \mathbf{u} \in \mathbf{N}_{0}^{k}$ существует многочлен $H \in J_{s-d}$ такой, что

$$
\left(G \mu_{d+1}\right)(\mathbf{u})=\left(H \mu_{d}\right)(\mathbf{0})
$$

и $\subseteq(H) \subseteq \mathscr{F}_{s-d}$. Тогда по предложению $7.4 H=\pi^{a_{s-d+1}-a_{s-d}} H_{1}$, где $H_{1} \in J_{s-d+1}$. Значит,

$$
\left(G \mu_{d+1}\right)(\mathbf{u})=\pi^{a_{s-d+1}-a_{s-d}}\left(H_{1} \mu_{d}\right)(\mathbf{0}),
$$

и так как по предположению индукции

$$
\left(H_{1} \mu_{d}\right)(\mathbf{0}) \equiv 0 \quad\left(\bmod \pi^{a_{s+1}-a_{s-d+1}}\right)
$$

To

$$
\left(G \mu_{d+1}\right)(\mathbf{u}) \equiv 0 \quad\left(\bmod \pi^{a_{s+1}-a_{s-d}}\right) .
$$

Вектор и выбран произвольно, поэтому $G \mu_{d+1} \equiv 0\left(\bmod \pi^{a_{s+1}-a_{s-d}}\right)$.

Пусть теперь $F \in J_{s-d}$. Из (C4) следует, что $F=K+\pi^{a_{s-d+1}-a_{s-d}} L$, где $K \in \mathscr{R}_{k} \chi_{s-d}, L \in J_{s-d+1}$. Тогда

$$
F \mu_{d+1}=K \mu_{d+1}+\pi^{a_{s-d+1}-a_{s-d}} L \mu_{d+1} .
$$

Согласно предположению индукции и уже доказанному утверждению (7.11),

$$
\mu_{d+1} \equiv \mu_{d} \quad\left(\bmod \pi^{a_{s+1}-a_{s-d+1}}\right), \quad L \mu_{d} \equiv 0 \quad\left(\bmod \pi^{a_{s+1}-a_{s-d+1}}\right)
$$

Значит,

$$
\pi^{a_{s-d+1}-a_{s-d}} L \mu_{d+1} \equiv 0 \quad\left(\bmod \pi^{a_{s+1}-a_{s-d+1}}\right), \quad F \mu_{d+1} \equiv 0 \quad\left(\bmod \pi^{a_{s+1}-a_{s-d}}\right)
$$

и для $i=d$ условие (7.12) выполнено.

Так как $J_{0}=I$, из (7.12) при $i=s$ следует, что $\pi^{n-a_{s+1}} \omega \in L_{R}(I)$.

Для завершения доказательства теоремы осталось показать, что $\bar{\omega}=\mu$. Из утверждения (7.11) следует, что $\bar{\omega}=\bar{\mu}_{1}$. Отсюда и из ранее доказанного соотношения $\bar{\mu}_{1}=\mu$, следует требуемое равенство.

Нормой последовательности $\omega \in R^{\langle k\rangle}$ назовем число

$$
\|\omega\|=\min \left\{\|\omega(\mathbf{i})\|: \mathbf{i} \in \mathbf{N}_{0}^{k}\right\} .
$$


Теорема 7.2. Если идеал $I$ имеет $K C O$ вида (7.8), то модуль $L_{R}(I)$ содержит систему последовательностей

$$
\alpha_{1}^{(t)}, \ldots, \alpha_{c_{t}}^{(t)}, \pi^{n-a_{t}} \alpha_{1}^{(t-1)}, \ldots, \pi^{n-a_{t}} \alpha_{c_{t-1}}^{(t-1)}, \ldots, \pi^{n-a_{1}} \alpha_{1}^{(0)}, \ldots, \pi^{n-a_{1}} \alpha_{c_{0}}^{(0)}
$$

таких, что последователвности $\alpha_{c}^{(s)} \in R^{\langle k\rangle}$ удовлетворяют условиям

$$
\overline{\mathscr{R}}_{k}\left(\bar{\alpha}_{1}^{(s)}, \ldots, \bar{\alpha}_{c_{s}}^{(s)}\right)=L_{\bar{R}}\left(\bar{J}_{s}\right), \quad s=0,1, \ldots, t .
$$

Любал тахал система последовательностей обладает такюе следующим свойством: если $\omega \in L_{R}(I) u\|\omega\|=b, n-a_{s+1} \leqslant b<n-a_{s}, s=0,1, \ldots, t$, то

$$
\omega \in \mathscr{R}_{k}\left(\pi^{n-a_{s+1}} \alpha_{1}^{(s)}, \ldots, \pi^{n-a_{s+1}} \alpha_{c_{s}}^{(s)}, \ldots, \pi^{n-a_{1}} \alpha_{1}^{(0)}, \ldots, \pi^{n-a_{1}} \alpha_{c_{0}}^{(0)}\right) .
$$

В частности, такая система порождает $L_{R}(I)$ как $\mathscr{R}_{k}$-модулъ, а ее параметры $c_{0}, \ldots, c_{t}$ можно выбрать так, что $c_{s} \leqslant\left|\mathscr{C F}_{s}\right|, s=0,1, \ldots, t$.

Доказательство. Пусть $s \in\{0,1, \ldots, t\}$. Заметим, что если $s=t$ и $\chi_{t}=\{e\}$, то семейство $L_{\bar{R}}\left(\bar{J}_{t}\right)=\{\overline{0}\}$ как $\overline{\mathscr{R}}_{k}$-модуль имеет единственную систему образующих $\{\overline{0}\}$. Пусть далее $s<t$ или $s=t$ и $\chi_{t} \neq\{e\}$. Так как множество $\bar{\chi}_{s}$ есть редуцированный базис Гребнера идеала $\bar{J}_{s}$, согласно теореме 7.1 множество импульсных последовательностей $E_{\bar{\chi}_{s}}$ мощности $c_{s}$ порождает $\overline{\mathscr{R}}_{k}$-модуль $L_{\bar{R}}\left(\bar{J}_{s}\right)$. По предложению 7.1 семейство $L_{R}(I)$ содержит систему последовательностей $\pi^{n-a_{s+1}} \mathfrak{U}_{s}$, где $\mathfrak{A}_{s}=\left\{\alpha_{1}^{s}, \ldots, \alpha_{c_{s}}^{s}\right\}$ - система последовательностей из $R^{\langle k\rangle}$, удовлетворяющая условиям $\left|\mathfrak{U}_{s}\right| \leqslant c_{s}, \overline{\mathfrak{A}}_{s}=E_{\bar{\chi}_{s}}$, и, в частности, обладающая свойством (7.14). Система последовательностей (7.13) составляется из подсистем $\pi^{n-a_{s+1}} \mathfrak{A}_{s}, s=0,1, \ldots, t$.

Рассмотрим произвольный набор последовательностей вида (7.13), удовлетворяющих условиям (7.14). Индукцией по $a=n-b=0,1, \ldots, n$ докажем (7.15). Если $a=0$, то $\omega=0$ и утверждение очевидно. Пусть $a>0$ и утверждение уже доказано для всех $\omega \in L_{R}(I)$ таких, что $\|\omega\|>n-a$. Рассмотрим случай, когда $\|\omega\|=n-a$. Тогда $\omega=\pi^{n-a} \nu$, где $\nu \in R^{\langle k\rangle}, \bar{\nu} \neq \overline{0}$. Так как $\omega \in L_{R}(I)$ и $a>a_{s}$, для любого $F \in J_{s}$ выполняется равенство $\pi^{a_{s}} F \pi^{n-a} \nu=0$, которое равносильно сравнению $F \nu \equiv 0$ $\left(\bmod \pi^{a-a_{s}}\right)$. Отсюда следует, что $\bar{\nu} \in L_{\bar{R}}\left(\bar{J}_{s}\right)$. Тогда для некоторых $b_{s 1}, \ldots, b_{s i_{s}} \in \mathscr{R}_{k}$ выполняется равенство

$$
\bar{\nu}=\bar{b}_{s 1}(\mathbf{x}) \bar{\alpha}_{1}^{(s)}+\ldots+\bar{b}_{s i_{s}}(\mathbf{x}) \bar{\alpha}_{i_{s}}^{(s)}
$$

и $k$-последовательность

$$
\varrho=\nu-b_{s 1}(\mathbf{x}) \alpha_{1}^{(s)}-\ldots-b_{s i_{s}}(\mathbf{x}) \alpha_{i_{s}}^{(s)}
$$

удовлетворяет условию $\varrho \equiv 0(\bmod \pi)$. Таким образом, справедливо неравенство $\left\|\pi^{n-a} \varrho\right\|=b_{1}>n-a$. Так как $\pi^{n-a} \nu, \pi^{n-a_{s+1}} \alpha_{1}^{(s)}, \ldots, \pi^{n-a_{s+1}} \alpha_{i_{s}}^{(s)} \in L_{R}(I), k$-последовательность $\pi^{n-a} \varrho$ также принадлежит $L_{R}(I)$ и для нее справедливо предположение индукции, т.е. $\pi^{n-a} \varrho \in \mathscr{R}_{k}\left(\pi^{n-a_{r+1}} \alpha_{1}^{(r)}, \ldots, \pi^{n-a_{r+1}} \alpha_{c_{r}}^{(r)}, \ldots, \pi^{n-a_{1}} \alpha_{1}^{(0)}, \ldots, \pi^{n-a_{1}} \alpha_{c_{0}}^{(0)}\right)$, где $n-a_{r+1} \leqslant b_{1}<n-a_{r}$. Из (7.17) следует, что

$$
\omega=\pi^{n-a} \nu=\pi^{n-a} \varrho+\pi^{n-a}\left(b_{s 1}(\mathbf{x}) \alpha_{1}^{(s)}+\ldots+b_{s i_{s}}(\mathbf{x}) \alpha_{i_{s}}^{(s)}\right) .
$$

Так как $b_{1}>b$, то $n-a_{r+1} \geqslant n-a_{s+1}$ и $s \geqslant r$. В итоге,

$$
\omega \in \mathscr{R}_{k}\left(\pi^{n-a_{s+1}} \alpha_{1}^{(s)}, \ldots, \pi^{n-a_{s+1}} \alpha_{c_{s}}^{(s)}, \ldots, \pi^{n-a_{1}} \alpha_{1}^{(0)}, \ldots, \pi^{n-a_{1}} \alpha_{c_{0}}^{(0)}\right) .
$$

Теорема доказана. 


\section{Список литературы}

1. Атья М., Макдональд И., Введение в коммутативную алгебру. Мир, Москва, 1972.

2. Бухбергер Б., Коллинз Дж., Лоос Р., Компъютерная алгебра. Мир, Москва, 1986.

3. Кузьмин А. С., Куракин В. Л., Нечаев А. А., Псевдослучайные и полилинейные последовательности. Труды по дискретной математике (1997) 1, 139-202.

4. Нечаев А. А., Конечные квазифробениусовы модули, приложения к кодам и линейным рекуррентам. Фундаментальная и прикладнал математика (1995) 1, №1, 229-254.

5. Adams W., Loustaunau P., An Introduction to Gröbner Bases. Amer. Math. Soc., Providence, 1994.

6. Becker T., Weispfenning V., Gröbner Bases: a Computational Approach to Commutative Algebra. Springer, Berlin, 1993.

7. Buchberger B., A note on the complexity of constructing Gröbner bases. Lect. Notes Comput. Sci. (1983) 162, 137-145.

8. Buchberger B., A critical-pair/completion algorithm for finitely generated ideals in rings. Lect. Notes Comput. Sci. (1984) 171.

9. Byrne E., Fitzpatrick P., Gröbner bases over Galois rings with an application to decoding alternant codes, J. Symb. Comput. (2001) 31, 565-584.

10. Cox D., Little J., O'Shea D., Ideals, Varietes, and Algorithms. An Introduction to Commutative Algebraic Geometry and Commutative Algebra. Springer, Berlin, 1998.

11. Jacobsson C., Lofwall C., Standard bases for general coefficient rings and a new constructive proof of Hilbert's basis theorem. J. Symb. Comp. (1991) 12, №3, 337-371.

12. Koblitz N., Algebraic Aspects of Cryptography. Springer, Berlin, 1997.

13. Kronecker L., Vorlesungen über Zahlentheorie. Teubner, Leipzig, 1901.

14. Krull W., Algebraische Theorie der Ringe. II. Math. Ann. (1923) 91, 1-46.

15. Snapper E., Completely primary rings. IV. Ann. Math. (1952) 55, №1, 46-64.

16. Kurakin V. L., Kuzmin A. S., Mikhalev A. V., Nechaev A. A., Linear recurring sequences over rings and modules. J. Math. Sci. (1995) 76, № 6, 2793-2915.

17. Kurakin V. L., Mikhalev A. V., Nechaev A. A., Polylinear recurring sequences over a bimodule. Proc. 12th Int. Conf. Formal Power Series Alg. Comb. FPSAC'00, MSU, June 6-30, 2000.

18. Landau S., Polynomial time algorithms for Galois groups. Lect. Notes Comput. Sci. (1984) 174, 225-236.

19. Mikhailov D. A., Polylinear shift registers and standard bases. Proc. 12th Int. Conf. Formal Power Series Alg. Comb. FPSAC'00, MSU, June 6-30, 2000.

20. Нечаев А. А., Линейные рекуррентные последовательности над коммутативными кольцами. Дискретная математиха (1991) 3, №4, 105-127.

21. Norton G.H., Salagean A., Strong Gröbner bases and cyclic codes over a finite-chain ring, Proc. Intern. Workshop on Coding and Cryptography. Paris, Jan. 8-12, 2001.

22. Sakata S., Synthesis of two-dimensional linear feedback shift-registers and Gröbner bases. Lect. Notes Comput. Sci. (1989) 356, 394-407.

23. Sakata S., A Gröbner basis and a minimal polynomial set of a finite $n D$ array. Lect. Notes Comput. Sci. (1991) 508, 280-291.

24. Sakata S., Two-dimensional shift register synthesis and Groebner bases for polynomial ideals over an integer residue ring. Discr. Appl. Math. (1991) 33, № 1-3, 191-203.

25. Samuel P., Zarisski O., Commutative Algebra. Springer, Berlin, 1979. 